\title{
LE PÈRE : UN HÉRITAGE ARCHAÏQUE ?
}

François Villa

\section{Presses Universitaires de France | «Revue française de psychanalyse »}

2013/5 Vol. 77 | pages 1381 à 1452

ISSN 0035-2942

ISBN 9782130618492

Article disponible en ligne à l'adresse :

http://www.cairn.info/revue-francaise-de-psychanalyse-2013-5-page-1381.htm

\section{Pour citer cet article :}

François Villa, «Le père : un héritage archaïque ? 》, Revue française de psychanalyse 2013/5 (Vol. 77), p. 1381-1452.

DOI 10.3917/rfp.775.1381

Distribution électronique Cairn.info pour Presses Universitaires de France.

(C) Presses Universitaires de France. Tous droits réservés pour tous pays.

La reproduction ou représentation de cet article, notamment par photocopie, n'est autorisée que dans les limites des conditions générales d'utilisation du site ou, le cas échéant, des conditions générales de la licence souscrite par votre établissement. Toute autre reproduction ou représentation, en tout ou partie, sous quelque forme et de quelque manière que ce soit, est interdite sauf accord préalable et écrit de l'éditeur, en dehors des cas prévus par la législation en vigueur en France. Il est précisé que son stockage dans une base de données est également interdit. 


\title{
Rapport de François Villa et discussions
}

\author{
Le père : un héritage archaïque ${ }^{1}$ ?
}

François VILLA

PRÉSENTATION DES HYPOTHÈSES ET DES AXES

Dans ce travail, je m'efforce de saisir comment se construit dans l'écrit freudien la figure du père et sa haute valence dans l'émergence de la vie psychique et du travail de culture. Plus qu'un retour à Freud, mon travail est un retour de ce qui, dans Freud, constitue l'énigme de ce père qui institue le sujet dans un rapport asymptotique à l'objet. Pourquoi le procès d'humanisation exigerait-il nécessairement de postuler, à son origine, le meurtre du père et le repas totémique qui s'en suit? Pourquoi ce meurtre, loin d'être accompli une fois pour toutes, reste-t-il pour chaque génération la tâche à accomplir pour que survienne l'individuation?

Aux commencements, il y a nécessairement du père et de la mère. Les processus de l'identification et de l'investissement libidinal d'objet s'accomplissent dans une contemporanéité rendant impossible leur distinction chronologiquement. L'identification primaire qui instaure la figure primitive du père aurait pourtant une antériorité logique sur l'investissement d'objet. En elle, résideraient les conditions du destin culturel des pulsions et de l'investissement psychique de l'objet. Les urgences de la survie imposeraient l'inscription du projet de la pleine satisfaction tant des exigences pulsionnelles que des souhaits inconscients dans un temps qui est celui de la contrainte vitale du différer (Derrida, 1967, pp. 293-340). De l'inachèvement,

1. En raison des contraintes éditoriales, ce texte ne reprend qu'en partie le rapport initial publié dans le Bulletin de la SPP $\mathrm{n}^{\circ} 106$ en octobre-novembre 2012 et il n'intègre pas les échanges auxquels il a donné lieu avant et pendant le cPLF. J'exprime ma gratitude à F. Benslama, D. Clerc, M. Girard, L. Kahn, P. Mérot, N. Minazio, E. Séchaud, A. Vanier, P. Valon, E. Weil, D. Widlöcher qui ont été mes interlocuteurs dans le cours de l'élaboration. Je remercie aussi G. Pragier et E. Chauvet pour leur attention et conseils. 
auquel est condamné ce processus d'identification entravé, naît une contrainte à le reprendre sans cesse pour le parachever et pour atteindre la volupté dans la jouissance de l'objet retrouvé perdu. Nous verrons alors comment il est possible d'avancer que le transfert est fondamentalement d'essence paternelle et de soutenir que ce sont les dérivations résultant du processus de transfert qui ouvrent l'historisation des vies individuelles, des groupes sociaux et de l'espèce.

Les motifs entravant l'identification primaire et son but premier rendent perceptibles que le procès culturel d'individuation prédispose aux procès névrotiques. La sauvegarde et le développement de la culture exigent jusqu'à un certain point la névrose (idéalement celle de transfert, mais sans pouvoir s'exonérer des autres formes psychopathologiques possibles). La névrose est donc le tribut, parfois exorbitant, que l'individu paye pour que son désir, auquel il ne renonce jamais définitivement, puisse exister au travers des déformations que sont ses réalisations mondaines.

S'affranchir de l'autorité paternelle ? Renoncer au père ?

En parlant d'entraves, je convoque forcément le complexe d'CEdipe et ses différents destins. Mes interrogations porteront, d'une part, sur les limites des humains à « surmonter » ce complexe et à s'affranchir des chaînes de l'autorité paternelle et, d'autre part, sur les fins du travail psychanalytique et du travail de culture. Peut-on concevoir une voie de résolution de ce complexe qui permette un réel affranchissement des chaînes de l'autorité paternelle et qui soit une alternative constituant une rupture de la contrainte à répéter le meurtre du père qui semble agir telle une fatalité ? L'enjeu de ces interrogations concerne et le devenir psychique individuel, et le devenir historique collectif. Ces devenirs sont inextricablement et indissolublement noués depuis ce que Freud désigne sous les noms énigmatiques d'héritage archaïque, d'héritage phylogénétique et dont il soutient la transmission, depuis la nuit des temps humains, de génération en génération. La Vatersehnsucht ${ }^{2}$ est, selon Freud, la force motrice du complexe paternel qui forme le noyau de cette acquisition phylogénétique. L'examen des conditions de possibilité de l'affranchissement

2. Sehnsucht a été traduit par nostalgie, aspiration, désir ardent, mais le mot allemand ne renvoie pas au temps passé comme la nostalgie, mais désigne l'aspiration à quelque chose qui aurait dû ou devrait être mais qui demeure absent et indéterminé. Les traducteurs des Euvres complètes propose désirance. Une expression de la Sehnsucht est le film de W. Wenders : Les Ailes du désir. Sur le rapport entre la Sehnsucht des romantiques allemands et celle de Freud, voir Vanier (2011). 
des chaînes de l'autorité paternelle inscrit le travail de la psychanalyse dans la suite de l'interrogation tragique d'Étienne de La Boétie (1576) sur la servitude volontaire.

Cette question de la servitude volontaire ne peut-elle pas se penser depuis l'expérience et la théorie du transfert s'organisant en néo-névrose dans la cure ? Le transfert et sa difficile « liquidation » sont-ils l'une des figures de cette servitude? Parmi les fins de l'analyse, n'y a-t-il pas cette visée d'affranchissement? Pourrions-nous soutenir que cette servitude volontaire n'intervient aucunement dans nos cursus de formation et dans le fonctionnement de nos institutions psychanalytiques?

Déplier la question paternelle permettra de mieux saisir les enjeux de notre pratique quotidienne de psychanalyste et son inscription dans le travail de culture. Il nous semble indispensable pour la théorie et la pratique psychanalytiques d'entendre ce qui oblige Freud à exposer sa découverte à la controverse avec d'autres champs de savoir (anthropologie, théologie, biologie...) à partir des concordances que constitue une certaine convergence d'objets. Pourquoi le fait-il en avançant des fictions théoriques qui n'hésitent pas, sans en faire fi, à s'inscrire, parfois, en totale contradiction avec les savoirs qui prédominent alors indiscutablement ? Par rapport à ce legs, notre projet est de discerner la nécessité métapsychologique et les déterminants de l'expérience clinique qui contraignent à une telle prise de risque théorique.

Un souvenir d'enfance de Léonard de Vinci (Freud, 1910 c, p. 148) dresse un constat: aujourd'hui, comme dans les temps originaires, rares sont les hommes qui peuvent renoncer au besoin de trouver un soutien auprès d'une quelconque autorité. Cette dernière renvoie toujours à la figure du père. Freud note que ce besoin est « si impérieux que [...] le monde se met à vaciller si cette autorité se trouve menacée ». Les hommes effectivement culturels sont ceux qui ont appris à se passer du père. Ce renoncement est la condition pour que la recherche déterminée par la curiosité sexuelle infantile ne soit pas entravée par la crainte du père en engendrant une inhibition de penser qui empêcherait le travail psychique de dérivation propre au travail de culture. Celui-ci est défini comme une activité de jugement (Freud, 1911 b) animée par un amour de la vérité dont la visée est de réduire la perte de réalité à laquelle l'homme n'est que trop porté par le principe de plaisir qui est au principe de la vie psychique (Freud $1924 e$ ).

Le risque du déplaisir provient, pour l'appareil psychique, de trois sources. D'une part, de la réalité de la vie pulsionnelle et du cortège des désirs inconciliables qui l'habitent; cette réalité confronte sans cesse à cette impuissance native qui est la marque de fabrique de l'activité psychique et se révèle 
structurellement insurmontable. D'autre part, de la réalité d'un monde qui ne veut rien (Winnicott, 1990 ; Gribinski, 2006) et dont les exigences font qu'à moins que nous ne soyons capables de nous modifier et de modifier le monde un tant soit peu, elle est aussi peu propice à répondre aux exigences de la pulsion qu'à satisfaire pleinement nos souhaits. Et, enfin, de la réalité du surmoi qui, paradoxalement, re-présente sans cesse au moi à la fois les exigences de la culture et celles du monde pulsionnel. Il relaie intrapsychiquement les exigences culturelles en témoignant du destin qui leur a été donné par chaque individu. Le surmoi est aussi le lieu où la contrainte de l'héritage archaïque se fait d'autant plus actuelle que, par défaut d'appropriation singulière, il est resté inactuel. Jusque-là, pour cet héritage, ne s'est présentée dans la pensée que la possibilité d'exister. L'activité de jugement se conclut par une décision dont le résultat est de parvenir à inhiber, plus ou moins, la pulsion quant au but sexuel direct et à détourner la curiosité sexuelle vers des activités culturelles - dont l'activité de recherche scientifique est le paradigme freudien - où elle pourra se développer avec hardiesse et indépendance. Freud soutient alors que « c'est dans le complexe paternel que nous reconnaissons la racine du besoin religieux » (Freud, 1910 c, p. 156). L'affranchissement des chaînes de l'autorité étant ce qui permettrait d'échapper à une vision pieuse du monde, d'instaurer la disponibilité à la culture.

Cette interrogation sur les destins du complexe d'EEdipe devra nous faire envisager qu'il n'est rien dans la structure de l'homme qui le prédispose à renoncer au père ${ }^{3}$. Nous reprendrons à notre compte la question de Nathalie Zaltzman à la fin de L'Esprit du mal :

«Les fils cessent-ils jamais d'être adossés à une référence paternelle qui, elle, certes, s'est considérablement modifiée, mais tend toujours, en dernier lieu, à se rétablir? Le progrès du travail de la culture, tel qu'il s'effectue à travers l'évolution œdipienne, qu'atteint-il au terme de son évolution, à quoi accède-t-il qui serait autre qu'un interdit sacrilège ?» (Zaltzman, 2007, p. 105).

La question du père présente dès les débuts

La psychanalyse « commence » par la question du père dans Die Traumdeutung $(1900 a)$ et elle « re-commence » sur les effets du meurtre du père sur la vie psychique et sociale des hommes dans Totem et Tabou

3. Paraphrase de ce que Freud écrivait à Binswanger : "Il n'est rien dans la structure de l'homme qui le prédispose à s'occuper de la psychanalyse. » Ces deux non prédispositions sont structurellement de même nature. Freud, Binswanger (1995, p. 134). 
(1912-1913 a) et L'Homme Moïse et la religion monothéiste (1939 a). Ces deux livres ré-interprètent l'enjeu du livre sur les rêves.

Dès la préface de 1909 au livre des rêves, le décor est planté. La mort du père est l'événement le plus significatif, la perte la plus radicale dans la vie. Dans l'ambivalence des sentiments que réveille la mort du père, dans le nécessaire accueil dans la conscience des éléments du conflit œdipien et dans l'incapacité à effacer les traces ${ }^{4}$, nous voyons poindre la nécessité de la mise en pièces du père qu'exige son dépassement et la nécessaire reconnaissance du meurtre accompli qui contraint la main à se retenir d'effacer les traces de l'action exercée par cet événement (Moscovici, 1991)

Totem et Tabou est le moment clé où la question du père prend sens depuis son meurtre. Ce forfait constitue l'acte $d u$ commencement, celui qui fait historiquement entrer l'humanité dans le procès d'individuation, dans le travail de culture. Mais il faudra attendre L'Homme Moïse et la religion monothéiste pour que la place nodale du père soit explicitée dans toute sa rigueur à partir de l'hypothèse de l'héritage archaïque.

Freud, le social et le politique : une « légende » tenace

Une « légende » tenace présente Freud comme uniquement soucieux de sa création, isolé du reste du monde dans son cabinet de travail, indifférent aux situations sociales et politiques. Il nous semble pourtant indéniable que ses travaux démentent cela. Marie Moscovici note que cette légende soutient également que Freud aurait tenu à l'écart de son travail la question juive et celle de son appartenance à ce peuple (Moscovici, 1986). Nous soutenons que les interrogations sur nos limites à « surmonter » le complexe d'CEdipe et à nous affranchir des chaînes de l'autorité paternelle sont devenues de plus en plus centrales pour Freud à partir, d'abord, de la Première Guerre mondiale et, ensuite, de la montée des totalitarismes à partir des années $1930^{5}$.

Ce que Freud écrit à ses différents correspondants à propos du Moïse montre clairement que son point de départ est, d'une part, la question du politique et, d'autre part, la question juive et celle de la récurrence millénaire de

4. Freud (1900 a [1899], p. 18 : «Après avoir reconnu cela, je me suis senti incapable d'effacer les traces de l'action exercée par cet événement »). Dans la deuxième partie du troisième essai de Moïse, Freud écrira : «Je n'ai pas été en mesure d'effacer les traces de la genèse de ce travail, qui fut dans tous les cas inhabituelle. »

5. Je partage cette lecture avec d'autres collègues : W. Granoff, M. Moscovici, N. Zaltzman, J. Puget, Y. Gampel, M. Viñar, P. Fédida, L. Kahn, M. Gribinski. 
l'antisémitisme. Pour Freud, l'étonnant pacte que le progrès a conclu avec la barbarie (Freud, 1939 a, p. 132) n'est pas contre nature, mais l'expression extrême de la tension insurmontable qui persiste dans les créations psychiques entre la survivance de la sauvagerie du primitif et le procès de sa transposition culturelle. Ces questions sont nouées fermement et inextricablement entre elles et à celle du destin individuel : celui-ci est impensable séparément du devenir de l'espèce.

\section{Idéalisation du père par Freud?}

Y a-t-il chez Freud un procès d'idéalisation du père ? La figure du père dans son œuvre n'est-elle fondamentalement déterminée que par son appartenance à un temps où prédomine toujours le dogme paternel (Tort, 2005) ? L'enjeu de l'entreprise freudienne est autre, il vise une déconstruction de la figure de la divinité pour dévoiler le procès de transfiguration par où le père est projeté de manière idéalisée dans les cieux (Freud, 1919 g, p. 214). L'expérience fait naître un doute et une inquiétude sur notre capacité à nous arracher au processus d'idéalisation du père, à sa transfiguration en figure divine ou protectrice. Nous sommes en présence d'une pensée tragique qui se confronte à la dure tâche, peut-être irréalisable, d'échapper au malheur destinal qui semble inhérent au complexe paternel. La levée du refoulement portant sur le forfait originaire peut-elle effectivement advenir et échapper à la logique de l'éternelle restauration du père dans ses droits dans le temps même où ils lui sont déniés ? L'expérience de la cure et de la vie sociale ne font-elles pas naître la crainte et le soupçon que cette logique relève soit d'une contrainte infrangible, soit d'une éventualité qui ne se réaliserait que fort rarement? Le rapport au père appartient-il psychiquement à ce qui peut connaître des modifications ou à ce qui s'avère inaltérable?

Une gêne technique

Avant de poursuivre, je mentionnerai la gêne technique éprouvée dans le traitement $d u$ père comme héritage archaïque. Je me suis retrouvé dans l'impossibilité de rédiger un texte de manière ordonnée et systématique. Je me suis vu contraint d'écrire des petits fragments dont chacun n'était qu'une variation autour du même thème. J'ai fini par me dire que la question du père ne pouvait s'aborder ni frontalement, ni d'un unique point de vue. Son traitement convoquerait inexorablement une forte tendance à la répétition 
qui s'exprime dans cette écriture en fragments. Chacun d'eux constitue l'un des éclats résultant de l'ambivalence à mettre le père en pièces et est un des lieux où s'accomplit le retour du refoulé : une des modalités de la résistance à l'accueillir. J'étais, à mon tour, sous le coup de la menace indiquée par M. Moscovici (1991, pp. 388-389) dans « Un meurtre construit par les produits de son oubli » :

«Il est vrai qu'il est particulièrement difficile de s'attaquer à cette question et de s'y tenir. De même que la métapsychologie, dans ses détours et ses détails, provoque en nous une sorte d'oubli constant, de nécessité de la ressaisir, de la réapprendre à chaque nouvelle lecture, la réflexion sur la phylogenèse a tendance à nous entraîner vers des généralités, à nous chasser de ce que l'on ressent comme le cœur des choses en psychanalyse, vers ce que l'on croit être sa périphérie, à nous détourner d'elle-même. D'apparence trop académique, ou à l'inverse impossible à penser, elle a au moins l'avantage de nous faire éprouver en nousmêmes, in vivo et au présent, la poussée du refoulement. »

Mythe ou (re)construction? Le pas à franchir entre le désir et l'acte

Reconnaître dans le meurtre du père une fiction théorique ne permet pas de la prendre à la légère, cela nous invite, en revanche, à penser que la structure du discours scientifique est fondamentalement de même nature que celle de la fiction. À mon avis, il est plus pertinent de désigner la fiction freudienne du nom de (re)construction que de l'assimiler à un mythe. Mythe et construction relèvent tous deux d'un travail. Mais, il nous semble que lorsqu'on parle du mythe, l'accent est mis davantage sur le résultat que sur le travail alors que c'est le contraire dans le cas de la construction. Celle-ci ne devient jamais une fin en soi, mais garde toujours la visée principale de lever l'inhibition du processus de remémoration. La reconstruction du meurtre a une portée fondatrice et elle met en œuvre l'étrange temporalité psychique mise au jour par la psychanalyse sous le nom d'après-coup.

Le lien entre Totem et Tabou et L'Interprétation des rêves est établi dans l'échange de Jones avec Freud après la terminaison du livre :

«Ses doutes étaient une simple réaction. Quand je le rencontrai, quelques jours après, lors d'un séjour à Vienne, je lui demandai comment il se faisait que l'auteur de la Science des rêves puisse maintenant douter de la sorte. Il répondit avec sagesse: "C'est qu'alors je parlais du désir de tuer le père et que maintenant je décris le fait réel ; après tout, il y a un grand pas à franchir entre le désir et l'acte" "(Jones, 1961, p. 377).

Cette réponse permet de cerner la différence entre mythe et construction psychanalytique. Le mythe accomplirait de manière déformée le désir, la construction psychanalytique viserait, en surmontant les inhibitions névrotiques, à ne pas esquiver la question de l'acte. De L'Interprétation à Moïse, le pas à franchir est effectivement celui qui existe entre le désir et l'acte qui vise 
à l'accomplir. Ce pas s'avère insuffisant et il reste à en faire psychiquement un autre. Celui-ci implique, dans un premier temps, de reconnaître que l'acte, bien que déjà exécuté, doit cependant être accompli à nouveau et comme si c'était pour la première fois et, dans un second temps, de réaliser psychiquement que l'on en est l'auteur. Chaque individu doit accomplir cette rude tâche qu'il ne saurait réaliser une fois pour toutes. Celle-ci impose de prendre acte de la survivance dans l'actuel d'un héritage archaïque qui fait des traits les plus primitifs les contemporains dans la vie psychique des formations les plus civilisées. Au cœur des réalisations culturelles les plus abouties, la sauvagerie des temps primitifs est psychiquement en acte : nous sommes les descendants « d'une lignée infiniment longue de meurtriers qui avaient dans le sang le plaisir du meurtre, comme peut-être nous-mêmes encore » (Freud, 1915 b, p. 151).

Des hommes qui s'entendent à mourir...

Nous prêterons attention à l'étrange déclaration faite dans la comparaison entre les attitudes, à l'égard de la mort et du meurtre, du primitif et du civilisé :

« Nous ne pouvons donc pas ne pas chercher dans le monde de la fiction, dans la littérature, dans le théâtre, un substitut aux pertes inhérentes à la vie. C'est là que nous trouvons encore des hommes qui s'entendent à mourir et, qui plus est, réussissent même à en tuer un autre » (Ibid., pp. 144-145).

Cette affirmation exige d'envisager que l'une des finalités du travail de culture et du travail de la cure serait qu'existent dans la réalité effective des hommes qui s'entendent à mourir et, qui plus est, réussissent même à en tuer un autre. Il ne faut pas entendre, ici, une apologie du meurtre, mais le projet analytique et culturel qu'adviennent des hommes dont la conscience s'étend jusqu'à la reconnaissance de cette dimension mortelle et meurtrière qui est au fondement de leur vie d'âme. Ils ne se déroberaient pas devant cette réalité sans pour autant tomber ni dans la substitution immédiate du faire au penser, ni dans cette procrastination qui caractérise la névrose de contrainte et qui se manifeste dans une pseudo-pensée qui remet le faire à toujours plus tard. Ils décideraient et accepteraient de différer la satisfaction pulsionnelle, d'accueillir dans la pensée le désagréable qu'engendrent les refusements ${ }^{6}$ de la vie, de supporter les modifications autoplastiques qu'impose la vie sans

6. Ce terme traduit celui de Versagung que l'on traduisait, le plus souvent, antérieurement aux OCF.P, par frustration. 
renoncer pour autant à modifier culturellement le monde et cet environnement dont ils supportent et reconnaissent la réalité et l'altérité radicale - à le modifier conformément, bien sûr, à leur désir.

Fondement et vraisemblance de cette construction

Ce qui inaugure la réflexion freudienne n'est pas le meurtre du père et la question paternelle, mais, avant tout, ces obstacles qui se dressent dans le cours de la cure psychanalytique. Ces derniers, soit, en compromettent la réussite, soit, nous obligent à faire preuve d'une réelle humilité quant à l'effectivité et à la pérennité des conquêtes qu'elle rend possibles. L'autre embrayeur de cette élaboration est le désenchantement du monde (Weber, 1964) qui surgit dans la vie quotidienne lorsqu'il devient évident que les progrès culturels accomplis sont loin d'être aussi grands que nous le croyons et bien moins acquis que nous ne le souhaitons. Dans L'Homme Moïse, obstacles et désenchantement se condensent dans l'énigme du pacte contracté entre le progrès et la barbarie et le mystère de l'antisémitisme. La tentative pour éclairer ces deux points est de comprendre l'étiologie de la religion monothéiste. Le rapport au père ne s'impose au principe de la vie de l'esprit au niveau de l'individuel et du collectif que chemin faisant. Et c'est la force heuristique de la fiction du meurtre du père qui pousse à la tenir pour un équivalent hautement plausible d'un processus sexuel agressif à portée traumatique : elle permet de construire une représentation qui serait l'asymptote d'un quelque chose qui, originairement, aurait pu arriver.

Dans l'héritage freudien, la méthode s'avère un legs plus essentiel que les résultats obtenus. Ces derniers ne sont le plus souvent que des constructions de secours, des échafaudages qu'il faudra prendre toujours garde à ne pas confondre avec la structure. Leur caractère asymptotique achoppe sur le fait que nous devons saisir la réalité à l'aide d'instruments qui sont de même nature qu'elle. La difficulté d'atteindre une adéquation de la pensée et de l'objet est structurelle et la méthode choisie détermine la construction de l'objet et détermine la forme de sa saisie.

Le modifiable et l'inaltérable. L'hypothèse controversée...

L'interrogation sur les limites du travail psychanalytique et du travail de culture devrait nous permettre de distinguer ce qui, dans la vie de l'esprit, relève d'une limite constitutionnelle spécifique et ce qui renvoie à une limite 
d'historisation. Nous reprenons à notre compte la distinction d'Aristote entre deux dimensions du réel quand il réfléchit sur la technique. Pour nous, travail de la cure et travail de culture relèvent d'une technique dont la mise en œuvre peut permettre à l'homme de changer son rapport au réel par modifications, transformations de la réalité antérieurement construite. L'œuvre de la technique permet de distinguer deux dimensions du réel pour l'action humaine. L'une lui résiste irréductiblement et, toujours, échappe à sa prise, on peut la désigner comme ce qui est et ne peut pas être autrement. L'autre ne lui résiste que d'une certaine façon et il peut avoir action sur elle : ce qui est mais peut tout aussi bien être autrement. Recourir à ces deux dimensions ne permet pas pour autant de parvenir à une distinction certaine. C'est pour supporter au mieux cette aporie que les psychanalystes ont posé, au principe de leur acte, la nécessité tant de l'analyse personnelle de l'analyste que de la pratique de la supervision des cures et de l'écriture « psychanalytique».

Adorno (1980, p. 63) avance que "de la psychanalyse, rien n'est vrai que ses exagérations », que celles-ci sont le vif même de la chose analytique. En accord avec cette proposition, je traiterai sérieusement l'exagération de l'hypothèse de l'héritage archaïque en me demandant ce qui serait et resterait vrai ou vraisemblable dans la construction freudienne d'un noyau constitué par les traces mnésiques héréditaires de l'événement historique que fut le meurtre $d u$ père. En quoi, la théorie paternelle freudienne lui est-elle intrinsèquement liée ? En prenant le temps d'entrer dans le paysage de la Vatersehnsucht que dresse L'Homme Moïse et la religion monothéiste, nous verrons la question du père prendre relief.

C'est à partir des analogies qu'il établit entre ontogenèse et phylogenèse que Freud avance l'hypothèse de cette survivance phylogénétique. Le complexe paternel avec la Vatersehnsucht qui l'habite sera désigné comme ce noyau de la vie psychique qui est une acquisition phylogénétique transmissible d'une génération à la suivante. La question du père que l'humanité s'est acquise par le meurtre devient le cœur de cet héritage archaïque qui, donnée constitutionnelle de la vie psychique, constitue sa prédisposition tant au travail de culture qu'à la formation des psychonévroses. Cette hypothèse est la seule qui permette, selon Freud, de jeter un pont entre la psychologie individuelle et la psychologie collective : les formations tant symptomatiques que culturelles sont alors entendues comme destin des traces laissées en chaque membre de l'espèce par cet "événement 》 fondateur. L'acceptation de ce postulat freudien comporte plusieurs questions. Cet héritage ne constitue-t-il pas, une part essentielle du refoulement originaire ? La pratique psychanalytique ne conduit-elle pas à se demander si elle n'est pas structurellement une part ineffaçable du programme de l'espèce 
humaine ? Serait-elle définitivement soustraite à toute prise de conscience ? Serions-nous condamnés à subir les effets toujours agissants de cet héritage qui serait la cause d'un inéluctable processus du retour du refoulé dont les destins s'exprimeraient dans les formes variables du malaise dans la civilisation?

\section{Transposition de l'individuel au collectif}

Freud espère comprendre, d'une part, comment la tradition efficiente ou active est présente dans la vie des peuple, quels sont les moyens dont se sert une génération pour transmettre ses états psychiques à la suivante et, d'autre part, les causes des manifestations résiduelles du travail psychanalytique (Freud, $1939 a$, p. 180). À partir du rapport au père, pourrait s'établir une parfaite concordance entre les commencements de la religion, de la moralité, de la société et de l'art et ce qui forme le noyau de toutes les névroses (Freud, 1912-1913 a, p. 377). C'est fort de l'acquis du traitement des phobies d'animaux que Freud se considère autorisé à mettre dans la formule du totémisme le père à la place de l'animal totem (p. 349). Cette transposition fait coïncider les deux commandements majeurs du totémisme : ne pas tuer le totem et ne pas posséder sexuellement une femme du même totem avec les deux désirs œdipiens qui forment le noyau de toutes les psychonévroses : celui de l'élimination du père et celui de l'inceste.

La mise en corrélation de l'interprétation psychanalytique du repas totémique et de l'hypothèse darwinienne de la horde primitive construit le socle de la fiction théorique du meurtre du père de la horde. Cette construction peut "paraître fantastique », mais elle a "l'avantage d'établir une unité insoupçonnée entre des séries de phénomènes jusqu'ici séparées »(p. 360) et elle rend possible une compréhension de la genèse et du développement du phénomène religieux et des symptômes. L'hypothèse prend la forme d'un conte :

«Un jour, les frères expulsés se groupèrent, abattirent et consommèrent le père et mirent ainsi un terme à la horde paternelle. Réunis, ils osèrent et accomplirent ce qui serait resté impossible à l'individu. (Peut-être un progrès culturel, le maniement d'une nouvelle arme, leur avait-il donné le sentiment de leur supériorité.) Qu'ils aient aussi consommé celui qu'ils avaient tué, cela s'entend, s'agissant des sauvages cannibales »(pp. 360-61).

Ce conte fait apparaître le rôle essentiel, dès les débuts de la vie psychique, de l'ambivalence (p. 377), comment s'imposa l'oubli du meurtre et comment la Vatersehnsucht put, à l'abri du refoulement, se renforcer en rendant inéluctable le procès du retour du refoulé.

À propos du retour de la fiction, vingt-cinq ans plus tard, nous ne suivrons pas Freud lorsqu'il écrit à Arnold Zweig (Freud, Zweig, 1973) que L'Homme 
Moïse contient une théorie de la religion qui n'apporte rien de nouveau par rapport à Totem et Tabou. La reprise de la fiction s'avère être, comme souvent dans la répétition, un retour où s'accomplit l'inévitable modification de ce qui fait retour ${ }^{7}$. Elle aboutit, à partir de la reprise de la vieille notion de série complémentaire et d'une réflexion sur la nature et la fonction de la période de latence, à une réĺlaboration de la théorie du trauma et à un approfondissement de la vérité historique approchée dans Totem et Tabou. La prise en considération de l'inéluctable processus du retour de refoulé contraint à penser que le meurtre du père ne relève pas d'un jadis révolu mais de l'actualité ${ }^{8}$ la plus vive de la vie psychique. S'accomplit également un développement de Psychologie des foules et analyse du moi (Freud, 1921 c) qui conduit à repenser le rapport entre la psychologie individuelle et la psychologie de foules sur d'autres modes que ceux de l'articulation ou d'un dualisme exclusif. Le temps de l'élaboration individuelle se conçoit alors dans sa résonance avec le temps de l'élaboration collective et depuis ces restes de la vie quotidienne dont la rencontre permet qu'après-coup l'infantile qui demeurait agissant advienne historiquement (Fédida, 1992, pp. 45-66) 9 .

Le crime n'a pas payé, le renforcement de la Vatersehnsucht

Le trauma, son oubli et la période de latence à laquelle met un terme le retour du refoulé jettent un pont entre l'individuel et le collectif et nous font penser que, du point de vue de la vie de l'esprit, névrose et civilisation relèvent d'une étiologie commune. L'ensemble de ce procès est la conséquence du fait qu'après la mise à mort du père primitif, aucun des frères ne s'autorise à franchir le pas qui verrait le triomphe de la psychologie individuelle du père de la horde (Freud, 1921 c, pp. 62-65). Chacun reste pris dans la psychologie de masse qui caractérise la foule indifférenciante des « frères ». L'un des effets du « renoncement » des frères est, par modification de la psychologie individuelle du père, la constitution, d'une psychologie individuelle limitée par les deux interdits dont elle prend source : celui de l'inceste et celui du meurtre. Chacun d'eux est la conséquence de la nouvelle position de sentiment qu'instaure la reconnaissance des dangers réels que comporte le désir de

7. Cette modification n'est pas immanquablement un progrès de la vie de l'esprit, mais peut parfois être une régression morbide.

8. À entendre au sens de caractère de ce qui est actuel, de ce qui tend à être en acte en ne restant pas seulement en puissance.

9. Ferenczi, (1982, pp. 139-147) désigne les restes diurnes comme des restes de vie. 
devenir père de la horde en lieu et place du père primitif. Le " renoncement » à aller jusqu'au bout de ce qui motivait le crime accroît considérablement, formations réactionnelles obligent, la Vatersehnsucht. La désirance à l'égard $d u$ père prend alors, manifestement, le dessus sur la haine et atténue l'intensité de la rébellion. Dans le retrait sur la personne propre, s'accomplissent des opérations psychiques dont le résultat se traduira, après-coup par une obéissance au père bien plus grande qu'elle ne le fut de son vivant. Aucun des meurtriers ne se substituera au père, mais chacun deviendra culturellement pour la première fois et dans le même procès, fils et frère : fils de celui qui, après le meurtre sera instauré, par la pensée et par une décision, comme père; frère en se reconnaissant comme tel par rapport aux autres auteurs du forfait. Les frères, pour des raisons de survie individuelle, contractent un pacte imposant l'exogamie et l'interdit du meurtre.

LES ENJEUX DE L’HOMME MOÏSE

Quelque chose qui serait arrivé : les motifs de L'Homme Moïse

Un faisceau de faits issus et de la clinique et, de l'histoire des civilisations conduit à la proposition que quelque chose serait arrivé qui a ouvert le procès historisant. Ce quelque chose est identifié comme le meurtre du père qui devient le prototype et le paradigme du procès de civilisation. Cette fiction vise à combler l'amnésie des débuts de l'humanisation : du point de vue de la remémoration, elle prétend, en raison de sa teneur en vérité, être un équivalent suffisamment vraisemblable de ce qui arriva. Ce quelque chose serait, à la fois, à l'origine du procès tant d'individuation que de civilisation.

Nous examinerons, d'abord, la proposition que le point de départ de L'Homme Moïse est la question politique et la question juive, puis nous soutiendrons que ce roman historique constitue une mise en œuvre du mode de penser psychanalytique : il est un authentique traité de pratique clinique.

Dans la « Remarque préliminaire I », écrite avant la décision de quitter Vienne, nous trouvons une déclaration tragique : «Avec la témérité de celui qui n'a rien ou que peu de choses à perdre, j'en viens pour la seconde fois à rompre une résolution bien fondée et à faire suivre les deux essais sur Moïse parus dans "Imago" de la partie finale tenue en réserve " (Freud, 1939 a, p. 56).

L'Homme Moïse doit être relu à partir de ce rien ou peu de choses à perdre qui fonde l'énonciation. Que faudrait-il ressaisir pour continuer, face à la 
barbarie triomphante, à penser et à agir ? C'est dans cette perspective que doit s'entendre le retour sur la théorie du trauma. Dans ce mouvement de lucidité aiguë, en septembre 1934, Freud écrit à Arnold Zweig (Freud, $1960 a$, p. 458) que c'est «à une époque de vacances relatives", alors qu'il ne sait « comment employer un excédent de loisirs » que Moïse s'invite et s'installe dans la maison de la psychanalyse. Il va habiter de manière insistante et toujours en suscitant l'ambivalence, les cinq dernières années de la vie de Freud. Moïse représente pour Freud l'inquiétante familiarité de l'étranger et aussi l'inquiétante étrangeté du familier. Il est la figure du retour, dans la psychanalyse, du rapport entre la grande et la petite histoire.

$\mathrm{Q} u$ 'est-ce qui fait histoire? Qu'est-ce qui fonde la construction historique? Que transmet l'histoire et comment le transmet-elle? Ces questions sont adressées tant aux temps immémoriaux des commencements qu'aux temps présents. Le sentiment d'urgence naît de ce qui se passe dans la vieille Europe que l'on croyait tellement civilisée que l'on en était venu à exclure, jusqu'à la Première Guerre mondiale, qu'y puissent fleurir ces comportements sauvages attribués aux peuples dits primitifs. Cette guerre entraîna bien des désillusions sur la réalité et la force des progrès accomplis par la civilisation, mais le monde ne savait pas que le pire était encore à venir. Un an et demi avant que Moïse devienne l'hôte ${ }^{10}$ de Freud, le 30 janvier 1933, Hitler fut nommé chancelier, les persécutions antisémites s'intensifièrent, le 10 mai eut lieu à Berlin le premier autodafé de livres : «En face des nouvelles persécutions, on se demande à nouveau comment le Juif est devenu ce qu'il est et pourquoi il s'est attiré cette haine éternelle ? » (Freud, $1960 a$ [1873-1939]).

Le retour de Moïse vient à la rencontre de ces questions qui, elles-mêmes, recherchaient les matériaux qui aideraient à les penser :

«J'ai vite trouvé la formule [pour répondre aux deux questions posées] : Moïse a créé le Juif, et mon livre a eu pour titre : L'Homme Moïse, roman historique [...]. Le sujet comportait trois parties, la première était intéressante comme un roman, la seconde était ardue et longue, la troisième substantielle et astreignante. C'est cette troisième partie qui fit échouer l'entreprise ; elle contenait une théorie de la religion qui n'a, à vrai dire, rien de nouveau pour moi après Totem et Tabou, mais qui apportait plutôt quelque chose de neuf et de fondamental aux non-initiés. C'est en pensant à ces non-initiés que j'ai décidé de tenir secret cet essai maintenant terminé. »

En lisant cette lettre, nous pourrions avoir le sentiment que l'actualité du pacte conclu entre le progrès et la barbarie s'estompe au point de faire

10. Les exils accomplis par ma famille m'ont rendu sensible à la richesse sémantique du mot « hôte » qui désigne tout autant celui qui donne l'hospitalité que celui qui la reçoit. Voir Benveniste (1969, pp. 87-101). 
oublier le bruit et la fureur qui déferlent sur l'Europe. Nous voilà invités à nous retrouver au moment de l'élaboration de Totem et Tabou en 1912-1913 avant même la sauvagerie de la Première Guerre. Face aux nouvelles persécutions qui désespèrent et confrontent à l'impuissance, que faire d'autre qu'essayer de s'accrocher à ses objets de pensée, à ses objets internes pour s'assurer que l'on n'est pas en train de tomber hors du monde (Zaltzman, 1998) ? Cette lettre exprime un mouvement de retrait des investissements du monde externe et un retour vers des investissements d'un moi d'un temps de paix (Freud, $1919 d$ ). Le moi est confronté à de l'impensable, à du terrible mais il n'a pas encore pleinement réalisé qu'il va se voir profondément modifié pour devenir le moi d'un temps où la plus folle destructivité régnera (Villa, 2011). Ce retrait est un de ces temps d'incubation ou de latence, si central pour la psychanalyse et dans Le Moïse, où la vie psychique peut, en hibernant, retrouver, après un certain laps de temps, les ressources d'élaborations.

Nous voilà, par la pensée, en 1913, à l'abri - si l'on veut. Dire qu'il n'y a rien de nouveau depuis Totem et Tabou est une déclaration qui ne manque pas d'ironie, car s'il en était ainsi pourquoi décider de tenir secret cet essai qui serait déjà terminé et cela alors même que son contenu est connu depuis déjà vingt-et-un ans? La dimension de roman historique peut conduire à sousestimer le «problème de nature principielle ou fondamentale ( Freud, 1939 a, p. 173) auquel tentent de répondre les trois essais et qui est constitué par un ensemble de questions. Comment se constitue la tradition ? De quelle dynamique paradoxale relève l'ininterruption et l'interruption de sa transmission? «Sous quelle forme est présente dans la vie des peuples la tradition efficiente ou active? » (Ibid). Comment participe-t-elle de la constitution subjective et quelle est son emprise sur un individu, sur un peuple?

Ces questions élargissent celle sur " comment le juif est-il devenu ce qu'il est? \(Freud, 1960 a [1873-1939], pp. 458-460). Leur traitement imposerait d'interroger la relation d'intense fidélité conflictuelle que le peuple juif entretient avec le monothéisme qu'il a inventé et qui constitue sa principale spécificité. Ce peuple, auquel il appartient, viscéralement et au-delà de l'entendement, devient alors, pour Freud, une figure exemplaire - d'autant plus questionnable «que de tous les peuples qui, dans l'Antiquité, ont vécu autour du Bassin méditerranéen, le peuple juif est pratiquement le seul à exister encore par le nom et sans doute également par la substance » (Freud, 1939 a, p. 183). Existe-t-il un lien entre l'aptitude à survivre face à l'adversité dont ce peuple a historiquement témoigné et ce que Freud nomme énigmatiquement la substance? Qu'est donc la substance d'un peuple? Qu'est-ce qui la détermine et comment se manifeste-t-elle effectivement en chacun de ses membres ? La substance est en étroite corrélation avec la constitution. Elle est ce qui 
constitue le support commun des qualités successives et ce qui est permanent dans un sujet qui est pourtant aussi susceptible de changer. Cette notion estelle un quasi synonyme de ce qui est désigné comme noyau de la vie psychique et de l'inconscient collectif?

Penser la nature de ces résistances est ce qui contraint Freud à postuler l'existence d'un noyau, principe actif de la vie psychique, contre lequel se dressent ces résistances psychiques qui peuvent entraver le travail de culture et s'opposer à ce que le retour du refoulé aboutisse à une levée effective du refoulement. Il se voit donc conduit à l'examen de sa nature, de ses fondements et de ses effets, et à s'interroger sur les pouvoirs et les limites du travail de culture et du travail psychanalytique.

Les manifestations résiduelles de la cure

C'est dans les manifestations résiduelles de la cure que devient indéniablement manifeste une force s'opposant tant aux progrès dans la vie de l'esprit qu'à la visée thérapeutique du travail psychanalytique. Dans le champ individuel, ces manifestations seront identifiées comme sentiment inconscient de culpabilité, masochisme originaire, réaction thérapeutique négative, excessivité du facteur quantitatif, bénéfices secondaires, autocratisme de la personne... Dans le champ social, on les reconnaîtra en tant que malaise inhérent à la civilisation, part d'hypocrisie propre au processus de culture, narcissisme des petites différences, propension volontaire à la servitude, contrainte à vivre individuellement au-dessus de ses moyens réels... Dans tout cela se manifeste, chez les individus et les peuples, leur peu d'appétit pour le changement, une volonté d'ignorer la vérité de la réalité psychique, la force incoercible des illusions engendrées, le besoin de croire. Ces résidus font naître le douloureux sentiment que travail de culture aussi bien que cure psychanalytique s'avèrent voués à échouer bien plus souvent que nous ne le concevons : les gains ne sont que moitié aussi grands que nous ne l'avions cru en les obtenant. En ce point, où semble s'échouer l'entreprise de culture et de la psychanalyse, s'exonde la force du primitif, de l'archaïque, de la destructivité dont il faut admettre la survivance agissante au quotidien.

Faire retour en 1913 n'est plus seulement une fuite devant les horreurs du présent, mais aussi l'espoir qu'à côté de la survivance en nous du primitif existent des ressources pour penser cette barbarie inédite qui commençait à régner. Avait-elle un lien avec la violence des temps primitifs (Villa, 2009 c), avec la haine craintive des fils envers le père et avec la tendance individuelle non seulement à la destruction, mais aussi à l'autodestruction que 
la cure psychanalytique oblige à prendre en considération ? La situation des années 1930 redonnait une tragique actualité aux questions apparues lors de la Première Guerre. L'expérience de la vie, celle de la cure obligeaient à s'interroger sur ce qui prédispose si peu l'homme au travail de culture et le rend, en revanche, bien plus apte à la névrose et aux régressions à des formes primitives des processus psychiques ainsi qu'à leur mise en acte dans les processus sociaux.

Depuis bien avant la Première Guerre, Freud est dans une intranquillité quant à la portée effective du travail psychique. Certes, celui-ci est capable de permettre à la vie de l'esprit d'accomplir de notoires et incontestables progrès, mais il est tout aussi indéniable que les digues qu'il dresse contre les risques de déferlement sauvage des forces pulsionnelles sont aussi fragiles que celles que les Hollandais ont érigées pour conserver les terres conquises sur l'océan. Ces conquêtes ont, certes, permis à l'humain de surmonter partiellement son impuissance native face à la chose sexuelle, mais il n'en demeure pas moins encore et toujours frappé du sceau de cette impuissance dont il continue à supporter durablement les effets. En conséquence de cela, il ne s'avère, à l'épreuve des nécessités de la vie, pas réellement apte à accomplir l'effort qu'exige ce travail psychique qui le rendrait un peu moins étranger, un peu plus ami avec la chose sexuelle qui le détermine et avec les vœux parricides et incestueux qui en forment le cortège. Il nous faut convenir que l'humain déploie, en revanche, de considérables efforts psychiques pour rester autant que possible identique à lui-même et cela au prix même de très grandes souffrances. Les réflexions d'Actuelles sur la guerre et la mort témoignent de cette préoccupation lorsqu'elles affirment que l'humanité ne vit jamais entièrement dans le présent, que dans la psyché le passé continue de vivre sans céder grand-chose aux influences du présent et en jouant un rôle puissant et peu déterminé par l'état présent du monde.

\section{L'Homme Moïse : un véritable traité de psychopathologie psychanalytique}

Jan Assmann (2001, p. 253) trouve déconcertant que, pendant les dernières années de sa vie, l'homme Freud, qui sait ses jours menacés, se consacre principalement à l'écriture de L'Homme Moïse et la religion monothéiste. Il se serait plutôt attendu à ce qu'il produise "une somme de sa théorie et de sa méthode " et non pas à ce qu'il s'aventure sur un terrain aussi controversé. Mais, c'est justement en affrontant la réactualisation du primitif dans le champ social que cet ouvrage constitue un compendium de la psychanalyse. Il est une réflexion sur le travail de la cure et sur le travail de culture, 
sur ce qui les permet, sur ce qui les entrave, sur les inévitables formations de compromis qui en résultent et sur leurs effets tout à la fois bénéfiques et maléfiques.

À la fin de sa vie, Freud est certes préoccupé par le destin de son Moïse, mais, pendant ce lustre, il écrira plusieurs écrits fondamentaux intimement articulés à ces trois essais. Début 1936, Un trouble du souvenir sur l'Acropole interroge également la question des conflits et des inhibitions déterminés par le complexe paternel. Au moment de la publication du premier essai de ce Moïse, L'Analyse finie et l'Analyse infinie opère un retour sur la question économique et sur la force du constitutionnel qui font partie de l'héritage archaïque (Freud, $1937 c$, p. 42) et qui obligent à s'interroger sur les limites qu'elles imposent au travail psychique et au travail de culture. Constructions dans l'analyse s'élabore après la publication du deuxième essai et met en œuvre le rôle, la nature et les enjeux, pour la technique psychanalytique, de la méthode de la construction dont L'Homme Moïse est un paradigme. Le troisième essai, achevé le 17 juillet 1938, s'accompagne de l'écriture du Clivage du moi dans le processus de défense qui étudie le rôle de ce mécanisme au cœur du fonctionnement psychique. Le Moïse à peine achevé, Freud met en chantier l'Abrégé de psychanalyse et Some Elementary Lessons in PsychoAnalysis où nous retrouvons tout l'apport sur l'héritage archaïque et sur les rapports entre l'appareil psychique et le monde extérieur. L'ensemble de ces textes est un legs dont les conséquences théoriques et techniques sont toujours actuelles.

Rappel de la trame de L'Homme Mö̈se et la religion monothéiste

Depuis Totem et Tabou, le meurtre du père est cet acte où se nouent inextricablement, dès les commencements, l'ontogenèse et la phylogenèse, il est à l'origine du complexe paternel. Celui-ci est le fond tragique de toutes les dramatisations permises par le complexe d'CEdipe et il forme la clé de voûte qui fait culturellement tenir les hommes ensemble dans cet équilibre déséquilibré qu'exprime l'inéluctable malaise dans la culture. Le troisième essai de L'Homme Moïse met à nouveau à l'épreuve cette conviction acquise un quart de siècle avant :

« Les phénomènes religieux ne [peuvent] être compris que selon le modèle des symptômes névrotiques de l'individu - qui nous sont familiers -, en tant que retours de processus significatifs, depuis longtemps oubliés, dans l'histoire originelle de la famille humaine, qu'ils doivent leur caractère de contrainte justement à cette origine et agissent donc sur les hommes en vertu de leur teneur en vérité historique » (Freud, 1939 a, p. 136). 
Le présupposé historique de cet essai s'étaye sur ce qui est historiquement établi à l'époque : la naissance égyptienne du premier monothéisme, l'abolition brutale de la religion d'Aton avec l'effacement quasi total des traces de son existence. À partir de là, l'auteur avance que Moïse était un Égyptien proche d'Akhenaton qui refusa la défaite du monothéisme qui mettait un terme à toutes ses espérances et à ses croyances fondamentales en le menaçant de devenir un renégat proscrit. Il se mit à la tête d'une tribu sémite exilée en Égypte depuis plusieurs générations qu'il élut comme son peuple dont il attendait le dédommagement pour ses pertes et la réalisation de ses idéaux. Il le libéra de ses chaînes en le faisant sortir d'Égypte, le sanctifia par la circoncision, lui donna ses lois et l'initia aux doctrines de la religion d'Aton. Cela ne se fit pas sans résistances de la part du peuple, d'autant plus que, dans un mouvement réactionnel consécutif à la défaite, Moïse avait durci les prescriptions d'Akhenaton. Face à la dureté de ces exigences religieuses, les Juifs se rebellèrent, tuèrent Moïse et rejetèrent sa foi. Après ce meurtre, le peuple poursuivit son exil jusqu'à Cadès où il s'apparenta avec d'autres tribus dont les Madianites et adopta une nouvelle religion vénérant Yahvé, dieu des volcans.

La période de latence

La notion de période de latence va s'avérer essentielle pour l'intelligence des processus de transfert et de transmission. Entre le meurtre de Moïse et l'installation d'abord à Cadès puis en terre de Canaan, Freud suppose qu'il a dû s'écouler un temps correspondant à, au moins, deux ou trois générations. Ce temps, nécessaire pour la compréhension historique, l'est encore plus du point de vue psychanalytique : les processus psychiques de l'oubli, du retour de l'oublié et leurs effets durables seraient sans lui impensables. L'histoire conduit à postuler que le triomphe sur Moïse n'avait pas pu abolir effectivement sa religion. Des traces et des impressions profondes en demeuraient qui, bien que méconnues consciemment, agissaient de manière latente sous une forme déformée dans les traditions qui se constituèrent. Avec le temps et avec l'aide de la culpabilité née de son assassinat, la force de la leçon de Moïse croîtra au point de faire retour sur le devant de la scène de la conscience et de l'histoire, d'entrer avec la religion de Yahvé dans un conflit dont la " solution » fut un compromis favorable à la doctrine de Moïse.

Les concordances établies entre les deux premiers monothéismes éclairent le fait remarquable que certaines traditions, loin de s'affaiblir avec le temps, voient leur pouvoir s'accroître en influençant de manière décisive les pensées, les actions d'un peuple, et de chacun des individus qui le constituent. 
Comprendre ces effets retardés est justement rendu possible par l'hypothèse de la période de latence et des processus qui s'y accomplissent. Le pari théorique est d'étendre à d'autres phénomènes de la vie de l'esprit et de la culture ce que la leçon psychanalytique a permis d'envisager comme l'étiologie vraisemblable des formations psychopathologiques. Ces phénomènes, incompréhensibles de prime abord, prennent sens en prenant en compte leur surdétermination qui les inscrit dans un processus historisant leur production. La période de latence abrite cette œuvre souterraine et conserve ce qui semble apparemment oublié alors qu'en réalité, il continue d'agir et de vivre de manière déformée au travers de nombreux éléments manifestes. Cette période s'installe à la suite d'une expérience de vie qui a pour effet de produire un tel bouleversement du point de vue économique, dynamique et topique que son premier destin ne pourra être que la réponse vitale que constitue son oubli. Mais, le processus d'oubli bute sur l'impossibilité de l'oubli qui se traduit par l'apparition de ce processus de retour du refoulé dont le caractère de contrainte ne cesse d'étonner. Périodiquement, l'oublié fait retour au point de vaincre, parfois, les forces qui rendaient amnésiques en mettant ainsi provisoirement un terme à la période de latence et en contraignant à la production de nouveaux compromis. L'instauration d'une tradition réside dans le compromis qu'elle permet entre l'oubli et son impossibilité : elle est le fossile vivant où s'exprime la restauration partielle de l'oublié dans son droit de cité, mais elle est aussi ce lieu où s'éteignent, par déformation, les revendications contraignantes du refoulé.

Pour Freud, la genèse des névroses humaines constitue l'unique analogie satisfaisante d'un autre effet retardé, elle lui paraît " très complète, elle s'approche de l'identité» (Freud, 1939 a, p. 151). Dans l'un et l'autre cas se rencontrent le phénomène de la latence, l'émergence de manifestations incompréhensibles, la condition de l'expérience vécue précoce, ultérieurement oubliée et le caractère de contrainte.

L'intellection des manifestations symptomatiques incompréhensibles n'est devenue possible qu'en tirant les leçons théoriques du traitement par l'hypnose. Son utilisation créait chez le patient un état d'inhibition des mécanismes de défense du moi favorisant le surgissement de pensées et de souvenirs oubliés ou n'ayant eu jusque-là que la possibilité d'exister. C'est à ces souvenirs et à ces pensées que se rattachaient en réalité les affects qui, faute d'avoir pu être traités, étaient restés « coincés 》 et avaient donné lieu soit à leur déplacement sur des représentations substitutives, soit à une conversion dans le somatique. Le rattachement des affects à ce qui les avait causés entraînait une abréaction adéquate qui avait un effet cathartique. La méthode et la théorie psychanalytiques procèdent de cette expérience qui conduit à postuler que tout symptôme névrotique actuel est le résultat d'un processus qui a commencé par une 
expérience de vie : quelque chose est arrivé qui a pu être plus ou moins traité psychiquement. Les symptômes sont l'indicateur de la qualification psychique donnée aux quantités d'excitation suscitées par l'événement. Ils sont à la fois le lieu de commémoration de cet événement et les fossiles où se produisent les effets présents ${ }^{11}$ de son insuffisant traitement psychique.

La méthode psychanalytique engage dans un mouvement de remontée du sens à partir du manifeste pour saisir les déterminants de la pensée et des processus qui le conditionnent. Dans ce travail se confirme structurellement que quelque chose a bien dû effectivement arriver et se vérifie également que nous ne savons pas et ne saurons peut-être jamais ce que $c e$ fut précisément. L'hypothèse il est arrivé quelque chose convoque ce travail de construction qui, sans atteindre à la réalité historique, en offrira un substitut restituant un morceau de l'histoire de vie perdue et ayant le même effet qu'un souvenir recouvré (Freud, $1937 d$; Villa, 1999). Le meurtre du père de la horde est cette construction qui, en raison du noyau de vérité qu'elle comporte, peut tenir lieu d'équivalent de la vérité factuelle insaisissable. Ce qui importe n'est pas que la construction corresponde point à point à cette réalité : sa pertinence réside dans le fait qu'elle permet au noyau de vérité qu'elle contient de laisser entendre ses effets malgré les déformations qu'elle a nécessairement imposées à la vérité factuelle.

Retour sur la théorie du trauma

À partir de la formule du développement de la névrose : «trauma précoce défense - latence - éruption de la maladie névrotique - retour partiel du refoulé » (Freud, $1939 a$,p. 159) est établi un rapport analogique entre le développement de la névrose (psychologie individuelle) et celui des phénomènes religieux (psychologie des masses). Ils ont pour ascendant commun le quelque chose qui est arrivé et expriment le sort qui lui fut réservé individuellement et collectivement. La reprise de la théorie traumatique commence par un rappel : "Les impressions vécues précocement, ultérieurement oubliées, auxquelles nous accordons une si grande significativité dans l'étiologie des névroses, nous les appelons les traumas (Ibid, p. 151)». Freud est attentif au malentendu qui peut naître de son affirmation qu'à l'origine de la névrose se trouve un événement historique. Conscient que cela pourrait être compris comme une réhabilitation de la vieille

11. Les fossiles ne sont pas seulement des vestiges pétrifiés d'êtres ayant vécu autrefois. Il ne faut pas négliger la présence d'un ADN en latence de vie et donc porteur de l'ensemble des informations héréditaires nécessaires au développement et au fonctionnement du vivant. Barriel (1997), L'ADN fossile, http://www.cnrs.fr/cw/dossiers/dosevol/decouv/articles/chap7/barriel.html. 
théorie du choc traumatique, il va déplacer l'accent de l'événement vers ses conséquences économiques dont dépend la caractérisation psychopathologique du processus. Ce n'est pas l'expérience en elle-même qui est traumatique mais les impressions qu'elle suscite et qui vont pouvoir, ou non, être traitées psychiquement. C'est le destin processuel de ce qui s'ouvre à partir d'une expérience vécue qui déterminera, après-coup, s'il y a eu ou non trauma.

Si les cures psychanalytiques nous ont appris que l'on ne trouvait pas systématiquement à l'origine de la névrose un événement exogène notoire, elles ont révélé qu'il y avait, en revanche, toujours : " une réaction extraordinaire, anormale, à des expériences vécues et à des exigences qui concernent tous les individus et qui sont élaborées et liquidées par eux d'une autre manière qui serait celle dite normale » (Ibid.). La conjecture est que, sans ces impressions très précoces, la névrose ne se serait pas déclenchée. Et, c'est pour échapper au retour sur le devant de la scène du faux dilemme sur l'origine exogène ou endogène des névroses qu'est convoquée la notion de série complémentaire ${ }^{12}$. C'est en soutenant que, dans toute névrose, est présente cette formule qu'il peut avancer que l'abîme entre les deux étiologies n'est pas infranchissable. À la série complémentaire est inhérente une autre conception du temps que celle de la flèche du temps. Freud distingue ce qui se passe aux extrémités de la série et ce qui peut émerger tout au long de la palette qu'elle dessine. Aux extrémités, agiraient essentiellement, voire exclusivement, soit la force et la nature du facteur exogène (névrose traumatique), soit l'augmentation endogène excessive de l'excitation qui emporte presque immédiatement la décision du choix névrotique (névroses actuelles). Pour comprendre la nature et les effets du trauma, il faut, entre ces deux bords extrêmes, introduire les notions de mémoire, de temps d'incubation ou de latence, de retour du refoulé et de l'après-coup. C'est l'ensemble de ce processus qui déterminera les formations individuelles et collectives résultant de l'accueil fait au retour du refoulé.

Cette réflexion dégage les caractéristiques que doit posséder le quelque chose qui arrive pour constituer un trauma individuel. L'expérience vécue doit appartenir à la première enfance (avant cinq ans) et elle a nécessairement un contenu sexuel-agressif suscitant un conflit d'ambivalence. Sa grande intensité psychique peut produire des endommagements précoces du moi. Il s'agit d'expériences vécues directement sur le corps propre qui, en raison de l'immaturité du petit humain, confrontent à de l'incompréhensible et laissent des traces des impressions sensorielles. Ces événements sont, en règle générale, totalement oubliés et inaccessibles au souvenir car ils tombent sous le

12. Introduite dans Freud (1916-1917 a [1915-1917], p. 360), cette notion n'est reconvoquée que dans L'Homme Moïse. 
coup de l'amnésie infantile. N'ayant pas pu être traités psychiquement, ils ne donnent pas lieu à un développement d'affect, celui-ci reste donc coincé. Après une floraison précoce, la vie sexuelle connaît, entre cinq ans et la puberté, une période de latence. Dans la névrose, les souvenirs qui agissent portent sur la période antérieure à la latence, celle de l'amnésie structurelle des débuts de la vie sexuelle. Amnésie et latence, qui caractérisent l'hypothèse de l'instauration en deux temps de la vie sexuelle de l'être humain, forment la condition effective de possibilité de la névrose. C'est de ce point de vue que la névrose peut être reconnue comme un privilège humain et comme une survivance des temps originaires (Freud, 1939 a, p. 54).

En raison de leur grande intensité psychique, les souvenirs oubliés acquièrent une force de contrainte qui défie les exigences du monde extérieur, n'obéit pas aux lois de la pensée logique et peut soit, cas extrême, imposer la substitution de la réalité du monde extérieur par la réalité psychique interne, soit, cas plus commun, entraver et inhiber significativement les activités psychiques. Dans tous les cas, la contrainte, qui résulte de la fixation au trauma, travaille au retour du refoulé. Dans la tendance à la répétition, Freud distingue les effets positifs et négatifs du trauma. Les positifs sont « des efforts pour remettre en vigueur le trauma, donc pour remémorer l'expérience vécue oubliée, ou mieux encore, pour la rendre réelle, pour en vivre de nouveau une répétition, ou, même si ce n'était qu'une relation d'affect antérieure, pour faire à nouveau revivre celle-ci dans une relation analytique à une autre personne » (Ibid.). Contre ces effets se dressent les effets négatifs qui sont des réactions de défense poursuivant le but opposé et allant de l'évitement aux inhibitions et phobies. Effets positifs et effets négatifs contribuent de manière déterminante à la formation de ces traits de caractère qui deviendront les constantes quasi immuables du moi (Villa, 2009 a). C'est du compromis entre les deux types d'effet que prendront forme les symptômes : témoignage à la fois du caractère insoluble du conflit et de la relative prédominance de l'une sur l'autre tendance dans le compromis.

L'éclosion de la névrose n'est pas toujours contemporaine de l'expérience vécue. Dans un premier temps, les conséquences de celle-ci peuvent, faute de symptômes manifestes, passer inaperçues. Il en est ainsi même quand le moi en construction a subi des endommagements qui pèseront structurellement sur son développement. Même dans le cas de la formation d'une névrose dans l'enfance, il est rare que celle-ci se poursuive telle quelle et sans interruption dans la névrose de l'adulte. L'instauration de la période de latence produit une accalmie du conflit où le développement semble se poursuivre sans notable perturbation jusqu'à l'irruption de la puberté qui sonne le réveil de l'originaire conflictualité psychique. Cette dernière peut s'intensifier jusqu'à faire 
entrer l'individu en profonde discorde avec les exigences culturelles du monde extérieur. Il n'en demeure pas moins que la névrose, forme pathologique du développement psychique, est aussi, et peut-être avant tout, une tentative individuelle, voire solipsiste, de guérison, elle est « un effort pour réconcilier de nouveau avec le reste du moi les parties de celui-ci séparées par clivage sous l'influence du trauma et pour les réunir en un tout exerçant sa puissance contre le monde extérieur » (Freud, 1939 a, p. 157).

L'aporie théorique et clinique que soulève Freud à la fin de ce développement rejoint les questions sur la possibilité psychique de s'affranchir de l'autorité paternelle. Oui, nous sommes effectivement en présence d'une tentative de guérison, mais, presque toujours, celle-ci, livrée à son propre cours, ne peut qu'échouer. Freud indique alors qu'elle a plus de chances de réussir quand le travail analytique vient à son aide, mais il concède que, même dans ce cas, elle peut se terminer très fréquemment « en une totale dévastation et un total éparpillement du moi, ou dans le terrassement de celui-ci par la partie dominée par le trauma, précocement séparée par clivage» (Ibid.). À la suite d'un cas clinique dont il dégage de la manière la plus épurée possible les enjeux sans entrer dans la présentation de ses singularités, Freud en vient à évoquer comment, avec le temps, la force de ce qu'il appelle le noyau de l'être acquiert une intensité telle qu'il impose sa contrainte au moi et peut conduire à des oppositions irréductibles au monde extérieur. Dans ce cas, l'identification du patient au père qui formait le noyau de son être avait connu de nombreuses vicissitudes sans jamais parvenir, au-delà de l'amour-haine pour le père, à un affranchissement des fixations premières. Avec le temps, le retour du refoulé entraîna la reviviscence de l'identification au père dans laquelle, en son temps, le petit garçon s'était engagé pour des motifs sexuels et le patient en vint à incarner une copie fidèle à l'image du père qui s'était formée dans son souvenir.

Conséquences collectives - traitement culturel du refoulement

À partir de la formule de la névrose : trauma précoce - défense - latence - éruption de la maladie névrotique - retour partiel du refoulé, nous sommes « invité[s] à faire le pas suivant qui consiste à admettre que dans la vie de l'espèce humaine s'est produit quelque chose de semblable à ce qui se produit dans la vie des individus » (Ibid.). Dans l'histoire de l'espèce, il y a eu aussi originairement des processus à contenu sexuel-agressif, qui sont oubliés en étant écartés défensivement mais dont les conséquences durables se font sentir après une longue période de latence. Ils sont à l'origine des phénomènes 
religieux qui sont, dans leur mode d'édification et leur tendance, semblables aux symptômes. Les phénomènes religieux et les phénomènes névrotiques relèvent d'un même événement survenu dans la famille humaine : les histoires du processus psychique d'individuation et du processus de civilisation vont de conserve depuis ce point d'origine commun.

En ce point, Freud ne revient pas, comme nous aurions pu nous y attendre, à la naissance du monothéisme juif en identifiant le meurtre de Moïse à ce processus sexuel-agressif originaire. Il nous convie à une remontée vers le temps des commencements de la civilisation en faisant appel au meurtre du père de la horde originaire. Avec ce saut dans la préhistoire de l'humanité, le meurtre de Moïse s'inscrit dans la temporalité, il est, dans le cours de l'histoire humaine, l'un des destins exemplaires du processus du retour du refoulé. Au moment même où la barbarie prétend briser l'unité de l'espèce humaine en mettant en œuvre la destruction de pans entiers de celle-ci, cette remontée dans le temps est un geste scientifique qui a une portée politique et éthique. Ce peuple juif, que l'antisémitisme nazi veut exclure de l'humanité en raison de sa supposée dégénérescence, se voit réinscrit, par L'Homme Moïse et la religion monothéiste, dans l'histoire du développement du phénomène religieux. Cette réaffiliation à l'espèce se fait au prix d'une désacralisation de ce peuple et de sa désendogamisation : peuple élu non par Dieu mais par Moïse, un homme étranger d'origine égyptienne qui lui transmit, voire lui imposa, après transformation, la religion monothéiste qui avait dominé l'Égypte pendant une courte période.

L'importance de l'exemple du peuple juif dépasse, pour Freud, l'appartenance à ce peuple que, bien sûr, il revendique. Dans le mouvement de l'apparition et de l'évolution des formes religieuses monothéistes qu'ouvre la religion d'Aton, son attention est retenue par l'opportunité de comprendre les particularités du monothéisme juif. D'une part, comment se fait-il que le peuple juif soit pratiquement le seul à avoir traversé l'histoire en conservant, non seulement, son nom, mais aussi en restant fidèle à une tradition d'une grande stabilité ? D'autre part, pourquoi ce peuple suscite-t-il au travers des siècles une telle haine meurtrière?

Pour Freud, le monothéisme naît comme réponse à un moment significatif du malaise dans la culture, celui où une " conscience de culpabilité croissante [s'est emparée] de l'ensemble du monde culturel de l'époque, en tant que précurseur du retour du contenu refoulé » (Freud, $1939 a$, p. 165). Ces questions deviennent une seule et même interrogation qui se décline sous différentes formes. Quel est le destin particulier que connaît le retour du refoulé dans le monothéisme? Que s'y accomplit-il qui serait absent des phénomènes religieux qui l'ont précédé ? En quoi se distinguent entre eux, quant aux résultats psychiques, les quatre monothéismes : celui avorté d'Aton, le juif, le chrétien et 
le musulman ? Le monothéisme n'est pas une affaire juive, pas plus que chrétienne ou musulmane. Il est l'affaire dont les humains ont à se saisir pour tenter de comprendre pourquoi l'illusion religieuse a pris une place si centrale dans le devenir de la civilisation et pourquoi elle semble encore promise à un grand avenir - en revêtant trop souvent les formes d'expression les plus destructrices. Le peuple juif, en raison de la forte contrainte à la fidélité qui a assuré la pérennité de ses traditions et de l'intense relation conflictuelle qu'il entretient avec ce monothéisme, prend une valeur paradigmatique. Il est, selon Freud, en tant que fossile vivant, le témoin parlant des effets du retour du refoulé.

La déconstruction du monothéisme par la psychanalyse vise à reconstruire le processus du retour du refoulé dont il résulte. C'est un essai pour penser les effets de contrainte qui s'exercent depuis ce refoulé et évaluer les forces dont disposent effectivement le travail de la cure et le travail de culture pour échapper, un tant soit peu, à l'inévitable compulsion de répétition et à l'inéluctable résurgence de la sauvagerie des processus primaires. Interroger les enjeux du monothéisme est donc l'occasion pour chacun de se demander en quoi il est encore, lui aussi, pieux (Nietzche, 1982, fragment 344). Pour ce faire, la réflexion freudienne accepte d'accueillir dans la pensée la question de savoir s'il y a, dans le psychisme, quelque chose qui relève du constitutionnel. Dès lors que la réponse est positive, il reste à se demander si la constitution peut être modifiée par les événements du présent et par le travail psychique. La conclusion qui s'esquisse, au terme du parcours freudien, est que cette entreprise s'apparente à une réelle aporie.

« AU COMMENCEMENT ÉTAIT L'ACTE »

Faire... penser !

Mais pourquoi, Freud a-t-il besoin de défendre, coûte que coûte, qu'il a dû réellement se produire un acte historique dont les caractéristiques principales, présentes dans la fiction du meurtre du père, seraient celle d'un processus sexuel-agressif ? Cette question est déjà abordée dans Totem et Tabou dont la phrase conclusive est : «[...] je suis d'avis, sans moi-même me porter garant de l'absolue certitude de mon option, qu'on est sans doute en droit d'admettre dans le cas que nous discutons : "Au commencement était l'acte" " (Freud, 19121913 a, p. 382). Le cas discuté est de savoir s'il est effectivement nécessaire 
" de ramener le début de notre bien-fonds culturel, dont nous sommes avec raison si fiers, à un crime odieux, offensant tous nos sentiments » (Ibid., p. 381) ou si, prenant en compte l'effectivité de la réalité psychique, nous ne pourrions pas nous contenter du seul dessein d'un tel crime. Freud remarque alors :

«Il n'est pas exact que les névrosés de contrainte [...] se défendent uniquement contre la réalité psychique de tentations et se punissent pour des impulsions seulement ressenties [...]. Il y a là aussi une part de réalité historique; dans leur enfance ces hommes n'avaient rien d'autre que les impulsions mauvaises, et dans la mesure où ils le pouvaient dans l'impuissance de l'enfance, ils ont aussi transposé ces impulsions en actions » (Ibid., p. 381).

Ce raisonnement permet de soutenir que :

«L'analogie des primitifs avec les névrosés est donc établie de manière beaucoup plus fondamentale si nous supposons que chez les premiers aussi la réalité psychique, dont la configuration est hors de doute, coïncidait initialement avec la réalité factuelle, et que les primitifs ont effectivement fait ce que, d'après tous les témoignages, ils avaient l'intention de faire » (Ibid., p. 382).

Mais, il ne faut pas, pour autant, méconnaître les différences entre primitifs et névrosés. Chez les premiers comme chez les seconds, «les démarcations tranchées entre penser et faire, telles que nous les traçons, ne sont pas présentes » mais « toutefois le névrosé est avant tout inhibé dans l'agir, chez lui la pensée est le plein substitut de l'acte. Le primitif est non inhibé, la pensée se transpose tout simplement en acte, l'acte est pour lui en quelque sorte plutôt un substitut de la pensée ».

L'enjeu de ce passage n'est rien de moins que les relations d'inhibition, de substitution et de plasticité qu'entretiennent entre eux, le faire et le penser. À l'arrière-plan de la comparaison entre primitif et névrosé se dessine en négatif une figure du normal. En pastichant le passage de La perte de la réalité dans la névrose et la psychose qui fait suite à l'assertion : " la névrose ne dénie pas la réalité, elle veut seulement ne rien savoir d'elle ; la psychose la dénie et cherche à la remplacer »(Freud, 1924 e, p. 39), nous pourrions la décrire ainsi :

«Nous appelons normal ou "sain", un comportement qui réunit des traits déterminés des deux réactions, qui, comme le primitif, ne se refuse pas au faire, mais qui, comme le névrosé, préalablement se contraint au penser. Ce comportement approprié à une fin, normal, conduit naturellement à une prestation de travail extérieure sur le monde extérieur et il ne se satisfait pas comme, dans la névrose, de l'instauration de modifications intérieures ; il n'est plus seulement autoplastique mais alloplastique. »

Ce que nous sommes appelés à concevoir est un penser qui, échappant à la menace de procrastination que comporte la croyance en la toute-puissance de la pensée, cesserait de se dérober éternellement devant l'acte et un faire qui ne serait ni un court-circuit immédiat de la pensée, ni un passage à l'acte, ni une répétition agie à la place de la remémoration, mais qui se soutiendrait 
d'une pensée qui réalise le passage du en puissance au en acte. Je n'ai pas innocemment pastiché le passage de La perte de la réalité : penser et faire sont ici des quasi synonymes de modification autoplastique et de modification alloplastique - ce qui ne nous surprend pas car, dès les débuts de la psychanalyse, il a été souligné que la motricité était le seul véritable indice de qualité pour distinguer réalité matérielle et réalité psychique (Villa, 2000).

Réalité psychique et réalité factuelle

Avancer l'existence d'un événement historique fondateur répond donc à la nécessité d'expliciter les raisons vitales qui contraignirent à sortir de ce temps originaire où la réalité psychique coïncidait initialement avec la réalité factuelle : temps du moi-ça indifférencié, temps du moi-idéal, temps du narcissisme originaire. Ce sont les urgences de la vie qui forcent à entrer dans cette complexification où s'invente l'appareil psychique. C'est excédé par la poussée qu'exercent sur lui les pulsions que l'humain réalise, pour la première fois, son absence de ressources appropriées pour faire face aux urgences de la vie dont il découvre alors la dimension contraignante. Plongés dans cet état d'Hilflosigkeit, il sera forcé de se rendre compte que, s'il veut recourir exclusivement à la voie des modifications internes, son entreprise est vouée à l'échec. C'est pour cela qu'il sera obligé d'emprunter une autre voie en se retournant vers le monde extérieur dans l'espoir qu'il pourra connaître une modification dont il pourra se saisir pour mettre fin à son état de détresse et parvenir à une satisfaction qui sera la plus proche possible de la pleine volupté.

L'état de détresse déterminé par les exigences des motions pulsionnelles convoque la nécessité d'un acte qui réponde de la pulsion en lui assurant un destin. Cet acte exige la sortie du solipsisme originaire et oblige à reconnaître l'existence d'un monde dont la réalité ne saurait se confondre avec la réalité à laquelle nous font aspirer les revendications pulsionnelles. Névroses comme psychoses sont des expédients ingénieux pour ne pas avoir à faire l'expérience des difficultés naturelles de la vie et pour échapper, autant qu'il se peut, à la reconnaissance des problèmes bien réels qui sont inhérents à la lutte pour parvenir à vivre en existant et à exister en vivant ${ }^{13}$. S'esquisse, ici, la figure idéale d'un individu qui ne se contenterait pas des seules modifications autoplastiques et qui ne renoncerait pas à accomplir des modifications alloplastiques, d'un humain dont le penser n'exclurait pas le faire et dont le faire se

13. Winnicott (1990, p. 108) : «Les plus grandes souffrances dans le monde des hommes sont probablement celles des personnes mûres, ou normales, ou en bonne santé. » 
soutiendrait du travail préliminaire du penser. Cela rejoint les préoccupations de Zaltzman concernant le travail de culture qui était, pour elle, un autre nom du travail de la cure et dans lequel elle voyait l'équivalent d'une instance de lucidité psychique résultant d'un processus d'élaboration intrapsychique et trans-individuel de l'expérience de vie qui modifie le développement individuel et l'évolution de l'ensemble humain.

Cet enjeu est d'une importance clinique essentielle. L'homme n'a-t-il pour seule alternative que de se détourner, par le refoulement, de la réalité psychique en se mettant au service de la réalité extérieure ou de refuser la réalité extérieure en se coupant d'elle et en restant au service des exigences pulsionnelles? Est-il envisageable que le travail psychique puisse permettre de se confronter aux exigences des pulsions avec lesquelles aucune véritable conciliation n'est possible et d'affronter les contraintes de la réalité sans s'y soumettre hypocritement? L'humain a-t-il les moyens psychiques pour faire face à l'impossible réel auquel le renvoient tant le monde dit interne que celui dit externe ? Peut-il lors du retour du refoulé accueillir effectivement ce qui fait retour ou est-il inéluctablement condamné à ce travail de défiguration qui rend, sous la forme d'un compromis, la chose acceptable pour la conscience ? Et peut-il, alors que rien ne l'y prédispose, parvenir, pour le moins, à une certaine aptitude à la modification qui oscillerait souplement entre autoplastie et alloplastie, et à un relatif va-et-vient fécond entre penser et faire ?

L'insuccès de l'acte : nécessité de distinguer interne et externe

Nous sommes donc à cet instant où il apparaît que, pour que survienne la modification interne qui témoignerait que l'état d'Hilflosigkeit a pu être surmonté, est exigée une modification du monde externe qui soit y fera apparaître l'objet du besoin (par exemple la nourriture), soit permettra de retrouver l'objet du désir inconscient (la mère comme premier choix d'objet), soit fera disparaître l'obstacle (le père) qui se dresse devant la satisfaction du besoin ou du désir.

Aux commencements du développement culturel et des processus d'individuation est donc posée la nécessité d'un acte. Nous noterons que si cet acte s'accomplissait et allait jusqu'à son terme (la pleine satisfaction), il n'y aurait nul commencement, mais un simple retour au degré zéro de l'excitation pulsionnelle, une extinction du processus. Il ne serait alors pas nécessaire d'avoir à conclure que la réalité psychique ne coïncidera en réalité jamais (si ce n'est pendant des instants éphémères d'illusion) avec la réalité factuelle et à se reconnaître condamné à une activité de pensée où la visée de l'identité de pensée se substitue à celle de l'identité de perception. Du point de vue radical 
de la satisfaction pulsionnelle, l'acte ne parvient jamais à son but premier et ultime et il aura toujours eu lieu en vain. L'insuccès de l'acte laisse en chacun des traces qui donnent lieu à un état d'affect: la Sehnsucht qui constitue le fond de l'âme humaine et forme sa prédisposition à la névrose. Il est la force motrice qui, depuis l'insuccès des incessantes tentatives de répéter l'acte pour qu'il aille, pour la première fois, jusqu'à son terme, engendre les formes polymorphes de substitution que nous reconnaîtrons comme l'histoire d'une vie.

L'événement historique que Freud postule aux commencements du psychique et du social découle de sa conviction que c'est une expérience vécue de Versagung qui contraint à la nécessité de distinguer l'interne de l'externe, la pensée du faire. Dans cette expérience, se découvre que le devenir de l'individu s'avère inextricablement noué au devenir du monde. D'une part, le monde extérieur devient le lieu où est faite l'expérience qu'il y a quelque chose dans la structure même des pulsions (de vie comme de mort) qui, constitutionnellement, se refuse à la pleine satisfaction. D'autre part, il se révèle que les satisfactions substitutives, que l'homme est capable de produire à partir du et dans le monde et qui tiendront lieu, tant bien que mal, d'équivalent de la satisfaction complète, dépendent de sa capacité à réaliser des modifications aussi bien auto qu'alloplastiques. L'épreuve du monde externe ne cesse de modifier, fondamentalement à son insu, l'individu qui, en se croyant le plus souvent identique à lui-même, ne cesse de tenter de modifier le monde pour l'adapter le plus possible à la satisfaction de ses désirs.

\section{Deux fictions à l'origine de l'acte : Sehnsucht et Vatersehnsucht}

À l'origine, la poussée pulsionnelle tente de s'accomplir dans un acte qui vise à modifier l'état du monde pour le rendre propice à la satisfaction alors qu'il ne l'est pas d'emblée. C'est en cela que l'acte est au commencement. C'est de l'échec de l'acte que naîtra la pensée et la voie de la compréhension mutuelle. Freud propose la fiction de deux scènes originaires (meurtre du père et expérience de satisfaction) qui figurent l'acte d'où émerge la succession de mécanismes psychiques de différenciation qui constituent le processus d'individuation. Les deux scènes montrent qu'un homme seul ça n'existe pas. Dans la scène de l'expérience vécue de satisfaction, sans l'intervention d'une aide étrangère ${ }^{14}$, ne pourrait pas avoir lieu la modification du monde permettant

14. Freud (1895, pp. 625-627). Dans cette scène, c'est l'aide étrangère (fremde Hilfe) et non l'être-humain-proche (Nebenmensch) qui est convoquée. La seconde figure n'apparait, logiquement, qu'ultérieurement après que, depuis les traces mnésiques de l'expérience de satisfaction, se 
à l'être en détresse de recourir aux moyens dont il dispose pour accomplir la modification interne qui supprimera la tension pulsionnelle. La scène du meurtre exige la coalition de ceux qui se sont vus imposer par le père ce régime drastique de privation sexuelle qui les a plongés dans un état permanent et vain d'excitation (état évoquant la névrose d'angoisse). Dans les deux fictions, l'association des individus entre eux n'est pas déterminée par une relation d'objet qui les relierait. Ce qui les unit n'est pas la sympathie causée par le partage d'un sentiment, mais la capacité primitive d'Einfühlung ${ }^{15}$. L'empathie est ce mécanisme primitif qui permet de percevoir dans l'autre (qui n'est pas nécessairement reconnu comme tel) une analogie significative, entre lui et l'autre, sur un point qui le plus souvent est la même aspiration qui se développe à partir d'un état d'affect.

L'Hilflosigkeit est un état de privation de satisfaction où l'humain découvre qu'il est privé des moyens pour faire face naturellement à l'excitation sexuelle. Le développement de l'excitation (expérience de la quantité), n'étant pas accompagné du développement d'affect (qui témoignerait de la qualification psychique de l'excitation), aura pour seul résultat le renforcement de l'intensité de la poussée exercée et la production d'affects coincés. Ce qui s'exprimera alors est cette Sehnsucht : état d'affect qui se traduit par une aspiration à une satisfaction encore jamais atteinte. La Sehnsucht est probablement présente dès avant les commencements. Elle est même ce qui y pousse et ce qui, demeurant toujours fondamentalement inaccompli, soumettra aussi à la contrainte de l'inévitable retour du refoulé. Hors le truchement que constitue autrui, le petit humain, livré à sa solitude, ne sait rien faire d'autre que transformer la Sehnsucht en angoisse. Cet état d'affect participe de la constitution de tous les humains et forme le terrain par excellence où peut s'exercer l'aptitude de l'appareil psychique à percevoir les déterminants inconscients de l'action de l'autre. C'est depuis cette communauté originelle d'affect que sont rendues possibles les communautés humaines. Cette Sehnsucht est à l'œuvre dans les deux scènes freudiennes des commencements. Dans l'expérience

développent les processus de remémoration et de jugement qui fondent la construction du complexe de l'êtrehumain-proche. La notion de Nebenmensch suppose l'introduction de l'individu dans les processus de reconnaissance permettant de poser autrui comme un semblable. Fremde Hilfe, moins anthropomorphe, convoque la dimension énigmatique de l'intervention de cet étranger qui peut s'avérer secourable, mais qui convoque au principe l'hostilité. S'entend, ici, la poursuite de notre discussion et de nos différences avec Mérot (2011).

15. Nous pouvons traduire par empathie si est maintenue la radicalité que lui accorde T. Lipps et qui est celle de Freud. L'empathie est ce mécanisme qui nous rend possible toute prise de position à l'égard d'une autre vie d'âme et qui nous permet un pouvoir se mettre ou un vouloir se mettre dans la même situation qu'autrui. Freud (1921 c, p. 45 et note 2, p. 48) et (1912-1913 a, p. 379). 


\section{2}

de satisfaction, elle permet la mise en association de la fremde Hilfe et du petit homme exposé à la privation. Dans l'autre scène, pour endiguer l'excessive excitation, elle met en masse ceux qui deviendront des frères après le crime. Une différence existe cependant entre les deux scènes. Dans la scène du meurtre, les auteurs du forfait sont, de manière contemporaine, dans le même état d'affect, c'est pour en sortir qu'ils se coalisent. Dans l'expérience de satisfaction, l'aide étrangère n'est pas, d'emblée, dans le même état d'affect que le nourrisson, mais elle dispose de la ressource de se remémorer cet état qu'elle a connu jadis : les traces mnésiques de ce ressenti la prédisposent à devenir une personne secourable.

L'hypothèse du retour permanent de la préhistoire dans l'histoire suppose qu'au cœur du psychisme agit la Sehnsucht dont la forme la plus radicale serait la Vatersehnsucht. D'abord, au fondement de la vie psychique individuelle et du travail de culture puis, toujours actuelle, elle œuvrerait au retour du primitif, du préhistorique dans l'histoire contemporaine. Elle est cette force désirante qui habite originellement chaque humain en engendrant un conflit d'ambivalence qui devient pérenne. Au for intime de chaque individu, existe, renforcée par le refoulement, la Vatersehnsucht qui constitue la communauté d'affect par où est rendue possible l'identification qui est au fondement de la formation en masse d'individus dépersonnalisés, mais unis par l'amour envers le même meneur et par la haine pour ceux qui sont identifiés comme les ennemis du père. Plus l'insuffisance d'élaboration de l'ambivalence sera importante, plus se fera sentir la nécessité de maintenir le refoulement et plus grand sera le risque que le retour du refoulé se traduise d'une manière prononcée par la dépersonnalisation de l'individu et par la haine envers l'étranger. La Sehnsucht, qui constitue le fonds impersonnel de toute personne, contraint sans cesse l'individu à réactualiser les premiers temps de son histoire. C'est lors du retour du refoulé que peut se prendre conscience des défenses employées jusque-là pour y échapper. L'élaboration psychique, en défaisant les défenses, peut permettre que l'histoire personnelle s'écrive depuis la reconnaissance du meurtre originaire : figure freudienne de l'Anankè.

L'acte originaire survient dans un processus d'hominisation qu'il infléchit en processus d'humanisation. Ses traces instaurent cet héritage spécifique qui comprend, en lui, la potentialité du retour de ce meurtre du père qui a été oublié ${ }^{16}$ et dont seront porteurs, nativement, tous les enfants de l'espèce. Cette scène originaire est celle de la première tentative d'affran-

16. Cette hypothèse éclaire les croyances populaires que les nouveau-nés seraient les revenants des ancêtres. Je reviens, plus loin, sur l'hypothèse du nourrisson comme réincarnation du père de la horde. 
chissement du père et celle de son relatif échec. Elle est celle de l'insuccès de la satisfaction totale et de l'engagement, à partir de ce revers, dans ces plaisirs substitutifs et ces petits arrangements qui permettent de rendre la vie supportable. Il faut accorder au meurtre une précession logique sur la satisfaction. Et cela, non pas parce que les enfants des hominidés non encore humanisés n'auraient pas connu la satisfaction, mais parce que la satisfaction de la scène éponyme ne peut s'entendre que du point de vue de l'invention du psychique. Cette expérience vécue (et pas seulement ressentie) de satisfaction ne prend sens que parce qu'elle met un terme à une expérience antérieure de douleur qui a convoqué la nécessité du recours à autrui et à l'invention de l'appareil psychique que son intervention permettra. L'expérience de satisfaction ne pourrait logiquement survenir et acquérir significativité que postérieurement à la scène du meurtre. C'est depuis la scène de privation originaire que se déploie cette poussée vers la satisfaction qui aboutira à l'insuccès dont les conséquences seront non seulement psychiques, mais fondatrices du psychique. L'expérience montre que les traces des insuccès sont bien plus contraignantes que celles des succès, elles poussent, inlassablement, l'inaccompli à s'accomplir. La scène de l'expérience de satisfaction est ce à quoi il faut se résoudre lorsque les nécessités de la vie ont contraints à renoncer à la satisfaction immédiate et directe qui était celle du père. Elle est le premier modèle de ces petits arrangements avec la vie par lesquels on se dédommage de l'échec de la première tentative d'affranchissement du père. Faute de pouvoir effectivement prendre la place du père s'ouvre la voie des transferts depuis la figure du père. C'est en cela que transfert est d'essence paternelle.

Le malaise indélogeable de ce qui a eu lieu sans être accompli

Du quelque chose, qui serait effectivement arrivé, nous ne savons donc consciemment plus rien, mais nous en subissons durablement les effets. Ce qui s'impose à Freud est que cela ne saurait être qu'un acte collectif qui engage l'aspiration la plus profonde de chacun des participants de l'ensemble qui l'exécuta. Ce quelque chose fait trauma et constitue une expérience vécue de nature sexuelle et agressive totalement oubliée dont il subsiste des traces sous la forme d'un endommagement non pas du moi d'un seul individu, mais des moi des individus qui ont vécu l'expérience. Subsistent aussi des restes de souvenirs isolés qui sont une survivance de l'expérience et qui œuvrent de manière déformée dans les mythes, les légendes, les fables, le folklore, les contes et les diverses ouvres culturelles. 


\section{François Villa}

L'aspiration profonde qui anime chacun est la source de la paradoxalité dont sont porteurs le psychisme et le social. Elle s'exprime dans ce malaise qui habite, tel un locataire interne indélogeable, au cour de la culture et de chaque homme et qui agit, répétitivement, comme une entrave au travail de culture. Cette aspiration, hautement singulière, la plus intime et la plus égoïste, est pourtant d'emblée ce qu'ont en partage l'ensemble des protagonistes. Elle constitue cette communauté d'affect originaire qui va faire des complices une masse où chacun est intimement liér à tous les autres au point d'être dans l'indifférenciation - voire dans l'impersonnalisation et cela, au moment même où, dans l'accomplissement de l'aspiration, se réalisait une opération de différenciation individuelle radicale où chacun était en train de se projeter dans le destin de l'Unique. Chacun ne s'aventure dans l'accomplissement collectif de cet acte que parce qu'il s'estime incapable de l'accomplir seul. Il s'y engage avec le violent désir d'en recueillir pour lui seul les fruits. L'acte constitue une épreuve de réalité où chacun des protagonistes se voit, pour des raisons d'autoconservation vitale, confronté, pour la première fois, à la nécessité d'un procès de renoncement découlant d'un acte de jugement. Le monde est certes modifié, mais il ne s'avère guère plus « propice » à la pleine satisfaction qu'il ne l'était antérieurement. Sous peine de mort est opérée une transformation du principe de plaisir. Le régime d'immédiateté est mis en échec dans l'émergence du principe de réalité où prédominent la logique et la nécessité du différer. Là où l'individu devait accomplir une suite de nouvelles actions lui permettant de recueillir pour lui seul les fruits de l'acte collectif survient un temps de suspension dont les précipités sont un ensemble d'opérations de différenciation psychique où s'effectue une prise de conscience de l'écart insurmontable qui existe entre ce qui est immédiatement attendu, espéré, désiré et ce qui peut être effectivement réalisé, obtenu, accompli. Se produit alors un retour réflexif sur la personne propre qui constitue le moment de naissance de l'acte du jugement : une pensée se substitue à l'acte, au faire. Dans cette suspension, qui est aussi mouvement de retrait de la pulsion, s'accomplit une rentrée de l'investissement libidinal projeté antérieurement sur le monde. L'effet de la contrainte externe force le moi-ça indifférencié à s'engager dans le processus de différenciation du moi d'avec le ça. Le procès engagé ne porte pas sur la seule différenciation du moi et du ça, il en enclenche d'autres : celle du couple en tension que représente le moi-idéal et l'idéal du moi (embryon du surmoi) et celle entre les narcissismes primaire et secondaire. L'un des principaux effets de ce procès de différenciation s'exprime dans la prise de 
conscience par l'individu de son existence et de l'existence d'autrui comme des existences radicalement séparées dans le temps même où, malgré la différence fondamentale, l'autre peut être aussi reconnu comme semblable.

Dans ce même mouvement de jugement est donnée une importance accrue à la réalité extérieure : elle est ce lieu où la pleine satisfaction est refusée, elle est le théâtre des contingences permettant l'accès au plaisir. L'homme prend conscience de l'existence de la réalité contraignante de ce qui, aspirant à s'accomplir pleinement, engage la vie comme intimement tressée à la mort. C'est quelque chose à quoi nous ne saurions véritablement renoncer une fois pour toutes, et à quoi nous allons apprendre à aspirer de manière non plus exclusivement immédiate, mais différée. Nous n'abandonnons jamais réellement le but premier, il reste inhérent à nos activités secondarisées qui tentent, en le reconfigurant, d'en endiguer l'action. Cela n'enlève rien à la valeur propre qu'acquièrent les buts secondaires : leur force est telle qu'ils entrent effectivement comme des constituants, bien réels, du conflit qui existe entre le but primaire et les buts secondaires dans le moi (Freud, $1940 e$ [1938], pp. 221-222). Ce moi, dès sa première effective différenciation d'avec le ça, se voit contraint à un mode paranoïaque de connaissance. Il doit rester, toujours, attentif tant à l'état dynamique du monde interne (rapport du moi aux exigences du ça et du surmoi) que du monde externe. C'est la condition pour que, si une motion pulsionnelle impossible à ajourner survenait à nouveau, il soit prêt à agir pour éviter que ne se réinstaure un état de détresse. Le moi est en état permanent de danger, même s'il est vrai qu'il parvient périodiquement à une mise en latence de cet état qu'il peut ainsi ignorer (principe de plaisir). Ce temps de suspens peut donner lieu à un acte de jugement : l'individu décide du destin qu'il donnera à la reconnaissance de la perception de son aspiration et des dangers réels auxquels elle l'expose. En ce point, Freud prend une position, qui est au fondement de la psychanalyse, en énonçant que la décision spécifique, fondatrice de la culture et du processus d'individuation, fut l'oubli de ce quelque chose qui est arrivé.

La Vatersehnsucht : une autre définition du « roc d'origine »

Dès les lettres à Flie $\beta^{18}$, est repérée dans les cures psychanalytiques, bien avant de pouvoir être nommée, la place nodale, pour l'étiologie des névroses, que joue l'ambivalence dans le complexe paternel. L'abandon de la théorie 
de la séduction déplace l'accent de la scène réelle vers le fantasme originaire de séduction mais le renoncement à l'hypothèse étiologique du père comme séducteur réel ne diminue en rien le rôle que joue celui-ci dans la formation des névroses. Nous pourrions dire que, bien au contraire, il acquiert, au-delà de la réalité factuelle de ce qu'il est et fait ${ }^{19}$, un rôle logique originaire d'embrayeur de la vie psychique dont il restera l'attracteur principal et l'essentiel organisateur. Du point de vue freudien, la mère est, dès les commencements, tout aussi présente que le père mais elle ne joue pas ce rôle nodal. Soutenir cela ne signifie pas minimiser l'importance de la relation primaire à la mère, mais souligner une différence : le conflit d'ambivalence ne lui est pas originairement inhérent.

La reconnaissance du rôle psychique clé joué par le père se poursuivra tout au long de la théorisation. Dans l'affrontement du petit Hans avec son père, Freud voit surgir in vivo le complexe paternel : la phobie se forme au point de rencontre de l'hostilité et de l'angoisse, là où le "souhait hostile réprimé » se transforme en peur du père. Avec le névrosé obsessionnel, est découvert à quel point ne pas s'affranchir de l'autorité paternelle contraint cruellement à devoir sans cesse servir deux maîtres tyranniques : un père ennemi impitoyable et un père adulé, l'un à immoler, l'autre à exalter qui se trouvent être en réalité une seule et même personne. Avec Schreber (contemporain de Totem et Tabou) est entraperçu que la tentative de guérison que représente la construction d'une théogonie privée s'origine dans le conflit d'ambivalence avec le père et s'ouvre la possibilité d'établir une analogie avec le développement des phénomènes religieux. L'intelligence de la fonction attractrice et organisatrice du complexe paternel (Ody, 1990) conduira à former l'hypothèse qu'il est un schème phylogénétique faisant partie d'un fonds psychique hérité.

Réalité factuelle, réalité historique et vérité historique

La reconstruction freudienne de l'histoire originaire nous confronte à la question cruciale soulevée en 1898 dans Les Souvenirs écrans : avonsnous à faire à des souvenirs $d$ 'enfance ou à des souvenirs sur l'enfance ? Probablement, n'avons-nous à faire qu'à des souvenirs sur l'enfance dont l'importance consiste dans le fait qu'ils accueillent un peu plus ou repoussent encore d'avantage l'infantile. L'infantile est un autre nom pour désigner ce quelque chose qui est arrivé sans avoir trouvé lieu psychique de représentation,

19. Pour qu'il n'y ait pas de malentendu : ce que le père est et fait dans la quotidienneté d'une vie n'est pas sans conséquences sur la vie de son enfant. Cela constitue les restes qui permettent à l'originaire de se réactualiser au présent. 
qui n'a eu que la possibilité d'être sans pourtant s'accomplir et qui se dérobe à tout souvenir (Fédida, 1995).

Le pont jeté par Freud entre la psychologie individuelle et la psychologie collective s'appuie sur les concordances (interdits du meurtre, du cannibalisme et de l'inceste) qu'il établit entre certaines parties des doctrines et rites religieux, et les formations névrotiques. Ces productions individuelles et culturelles témoignent, tout à la fois, du respect de ces interdits et de la rébellion contre eux. Elles sont le lieu paradoxal d'une survivance au travers, d'une part, de fixations à l'ancienne histoire familiale où prirent naissance, dans un procès ambivalentiel, les interdits et, d'autre part, de restaurations du passé à la suite des retours, après de longs intervalles, de l'oublié. La fiction freudienne, en déconstruisant ces fixations et restaurations, remonte psychiquement le cours de l'histoire jusqu'en ce point où se laisse entendre la vérité historique. Celle-ci n'est ni la réalité psychique, ni la réalité matérielle. La réalité psychique n'est pas une réalité individuelle et purement personnelle; elle se constitue et se développe à l'épreuve d'une réalité matérielle (elle-même construite) qui lui résiste et déjoue la possibilité d'établir par la perception une identité durable entre les deux réalités (sauf psychose ou coup de foudre). La vérité historique désigne ce quelque chose qui, en nous re-confrontant à de l'impossible, provoque une solution de continuité entre les deux réalités. La teneur en vérité d'une (re)construction tient dans son pouvoir de réactualiser ce quelque chose dans une figure dont l'efficience permettra de penser que, si elle n'est pas la chose même, elle est, cependant, un équivalent qui peut en tenir lieu de manière suffisante et qui peut donc être considérée comme une conquête de la vie de l'esprit.

Ce sont les fixations et restaurations présentes dans les doctrines et rites religieux qui permettent d'envisager la plausibilité de la présentation freudienne de l'histoire originaire de la culture et de l'individuation. Cet ancrage sur le sol de la vérité historique, qu'il ne faut pas subsumer sous la réalité historique, nous force à convenir que cette fiction du meurtre du père garde une réelle portée heuristique et possède une force probante indéniable. La scène des commencements tout autant que le rôle du père dans la procréation sont un sujet de doute que nous devons supporter et qui va constituer le ferment actif de la pensée. La fiction freudienne n'est pas, comme le propose Lévi-Strauss, un mythe de plus (ce qui serait déjà énorme), mais le lieu où se laisse entendre ce qu'est l'opérateur de tout mythe et ce qui rend possible une histoire de l'humanité. La notion de complexe paternel oblige la pensée à envisager individu et collectif comme un continuum sur lequel peuvent se produire, contingences de la vie obligent, des effets de singularité (Villa, Weil, 2011) et elle nous invite à explorer les processus de transposition qui s'opèrent ou non entre eux. 
Naissance de la communauté de culture

L'émergence du monothéisme représente l'un des destins de ce qui a " concerné tous les hommes originaires » (Freud, 1939 a, p. 160) et qui ne s'est pas produit en une seule fois. La reconstruction de la genèse du processus se révèle être, fatalement, un travail de réduction qui s'exprime dans la facilité de langage qui conduit à dire : « un jour, les frères expulsés... ». L'histoire, en réalité, s'est étendue, écrit Freud, sur des millénaires pendant lesquels le quelque chose s'est répété un nombre incalculable de fois avant d'acquérir, pour une première fois qui ne saurait être la dernière, sa significativité psychique et sa portée culturelle. Avant que le meurtre ne donne naissance «à la première forme d'organisation sociale bâtie sur le renoncement pulsionnel », il faut envisager une longue période où les frères entrent en guerre entre eux parce que chacun veut être le seul à détenir la totalité des prérogatives du père. Une longue suite de meurtres s'ensuit qui prend fin dans la prise de conscience des dangers que fait encourir une telle prétention d'exclusivité, dans la reconnaissance de l'insuccès auquel elle est vouée et dans la décision qui en résultera.

À partir du totémisme se produit une évolution vers l'humanisation de l'être vénéré. En lieu et place de l'animal totémique, apparaissent les dieux humains qui restent, sous diverses formes, apparentés à l'animal totémique. Pour Freud, dans cette évolution vers l'anthropomorphisation de la divinité, il y a probablement eu une précession des divinités féminines et principalement maternelles sur les divinités masculines et paternelles, et une antériorité du droit maternel sur l'ordre paternel. L'instauration de celui-ci s'exprime dans l'apparition de nouveaux pères qui n'atteindront jamais la toute-puissance du père de la horde : il n'y aura plus un père unique, mais plusieurs pères qui se voient obliger de partager leurs prérogatives en contractant un pacte leur permettant de vivre ensemble sans constamment s'entretuer. Le pas suivant met un terme au polythéisme et signe le retour de l'oublié, par l'invention du monothéisme, d'un seul et unique dieu-père à la puissance illimitée et qui fait montre d'intolérance meurtrière à l'égard de tout autre dieu (Assmann, 1999). L'hypothèse de la tendance à la restauration du passé qui impose, après des temps de latence plus ou moins importants, le retour du refoulé sur le devant de la scène tant individuelle que collective forme la contribution décisive de la psychanalyse à la compréhension du développement historique du phénomène religieux. Une des singularités les plus marquantes de cette tendance est que «tout morceau faisant retour de l'état d'oubli s'impose avec une puissance particulière, exerce sur les masses humaines une influence incomparablement forte et prétend irrésistiblement à la vérité, face à laquelle une objection logique 
reste impuissante. Ce curieux caractère ne se comprend que selon le modèle de la folie délirante des psychotiques » (Freud, 1939 a).

Imaginons, maintenant, ce qui, pour chacun et pour l'ensemble des frères, se passe et les empêche d'aller individuellement jusqu'au but visé par le meurtre. Dans Psychologie des masses, il est indiqué que c'est le père de la horde qui, par son égoïsme et son intolérance sexuelle, " contraint pour ainsi dire [les fils] à rentrer dans la psychologie des masses ». Empêchés par la force du père de satisfaire leurs tendances sexuelles directes, ils se retrouvent plongés dans un état de privation qui les contraint à entrer dans les prémices d'un lien social. La coalition des frères ne vise pas à changer l'organisation de la horde, chacun des conjurés escompte recueillir les bénéfices du forfait en s'installant à la place du père. Il est vraisemblable que, dans un premier temps, l'un des coalisés, malgré les menaces qui pèsent sur sa vie, tente de s'octroyer cette souveraineté du père qui fait coïncider la puissance et le pouvoir ${ }^{20}$. La conséquence pour lui est de connaître, tôt ou tard, le sort du père. C'est à la suite d'un certain nombre de répétitions du meurtre qu'aucun des fils n'assume plus, pour des raisons d'autoconservation, de céder à la tentation d'occuper cette place et se résout à entrer dans une société « égalitaire 》. Mais le recul des fils laisse une place vide par où va, d'abord, s'accomplir le retour du père et de l'idéal controversé qu'il représente et, ensuite, s'accomplir sa restauration dans les droits que la coalition lui a contestés. Là, va s'effectuer la transfiguration du père (Benslama, 2003, pp. 139-148; 2004, pp. 93-102) : d'abord, par l'apparition du totémisme et des tabous qui lui sont afférents, puis, par la projection en divinité de la figure transfigurée. Le totem est le substitut du père et les tabous le résultat de l'extension des propriétés du père aux femmes dont il avait jadis la possession sexuelle. Le dieu est cette forme plus tardive qui résulte de la décomposition du totem et de la déconstruction de l'ambivalence. Dans un premier temps, le totem condense plastiquement deux strates historiques : celle du meurtre du père et celle, logiquement postérieure, de sa réinstauration dans ses droits. Pour Freud, le fait que, dans les mythes, le dieu apparaisse comme participant, au côté des frères, à la consommation de l'animal totémique sacrifié est le résultat d'une séparation - d'un

20. Sur la souveraineté, Girard (2012), Expériences de la communauté. Hétérologies politiques de Georges Bataille. 
clivage - entre ces deux temps. L'animal représente une défiguration du père sur laquelle se portent les sentiments hostiles, la haine jalouse - le maintien de l'animalisation du père témoigne que les sentiments agressifs exigent encore et toujours le refoulement. Mais, le renforcement des motions de sentiments tendres du fils envers le père permet le déplacement d'où s'engendre Dieu comme figure anthropomorphe. C'est peut-être à partir de ce recul des fils que les hommes ne savent plus ni mourir, ni donner la mort.

Transfert depuis le père vers la mère

L'infléchissement du dessein initial s'accompagne d'un changement de position par rapport aux femmes de la horde dont le père avait la jouissance exclusive et d'un extraordinaire accroissement de la Vatersehnsucht. Au moment où chaque individu, sous peine de se voir assassiné à son tour, est contraint de renoncer à l'aspiration d'occuper la position du père, s'impose logiquement une distinction entre les femmes de la horde. En prenant en horreur l'acte accompli, en le regrettant et en l'oubliant, chacun des coalisés régresse à la position antérieure de fils soumis au père par la crainte. Il ne s'agit pas de la simple restauration d'un état antérieur, mais plutôt de l'émergence d'un état nouveau où les femelles de la horde vont être distinguées comme permises ou interdites. Certaines vont acquérir le statut de mère et de sœurs qu'instaure l'interdit d'un commerce sexuel endogame : on renonce à les posséder sexuellement. Dès lors ne sont plus convoitables et permises que les femelles qui ne sont pas les « véritables » objets initiaux du désir (les femmes que possédaient le père). Ce nouvel état de fait force à accomplir un travail de déplacement du désir de l'objet véritable vers des objets qui, sous certaines conditions, seront tenus, du point de vue de l'identité de pensée, pour des équivalents.

Freud note qu' " une bonne part du pouvoir absolu libéré par l'éviction du père passe aux femmes, ce fut la venue du temps du matriarcat ». C'est en prenant appui sur la surprenante notation qui clôt l'article d'Andréas-Salomé (1928) intitulé « Ce qui découle du fait que ce n'est pas la femme qui a tué le père »: " tout passe par la veuve du père » que nous entendrons cette remarque. De la place laissée vide par le meurtre émane maintenant une aura, une autorité dont l'ombre tombe sur toutes les femmes que possédait le père de son vivant : un interdit de les convoiter les transforme en veuves du père symboliquement liées à lui. Une partie de la force, du pouvoir qui était l'apanage du père se transfère aux femmes. En tant qu'anciennes possessions sexuelles $d u$ père elles en deviennent les porteuses, les représentantes. La crainte, le respect, l'amour, l'ambivalence qui constituaient le lien au père sont déplacés sur 
ces objets sexuellement convoités. Sur eux est transférée la figure du père, ils inspirent alors le respect qu'imposait le père. Les fils se tiendront désormais, à nouveau, à une distance respectable de ces objets. Ils redouteront de se trouver dans la proximité des femmes désirées comme ils craignaient, hier, pour des raisons différentes mais analogues, d'être dans une contiguïté qui les mettait à la portée de la main du père ${ }^{21}$. Dans la peur masculine de la femme survivrait et s'exprimerait la peur de rencontrer en elles le père, sa colère, sa puissance et sa vengeance. Les femmes participent ainsi de la transfiguration du père, de sa sacralisation et de son élévation divine, elles deviennent porteuses dans l'actuel du phallus du père - des déesses mères parées des attributs de la toutepuissance du père de la horde.

\section{La communauté des frères}

Pourquoi la situation instaurée par l'élimination du père contient-elle un facteur engendrant, dans le cours du temps, un extraordinaire accroissement de la Vatersehnsucht? Le désir de devenir semblable au père en restant inaccompli entraîne avec le temps une atténuation de la rancœur envers le père qui est contemporaine de l'apparition d'un idéal du moi dont le contenu est conflictuel : désir d'avoir entièrement la puissance du père et, en même temps, désir de se soumettre à lui. Entre les pères que pourront devenir les fils rebelles et cet idéal du moi subsiste un écart où se maintient, agissante, la Vatersehnsucht.

Pourquoi, dans la société des frères, la rancœur envers le père s'atténueraitelle ? Est-ce parce que sa mort fait que ce n'est plus lui qui, dans la réalité matérielle, barrera la route de la satisfaction ? Il est toujours présent, mais il a subi une dématérialisation par le transfert de ce qu'il représentait, d'une part, à des instances psychiques et, d'autre part, à des institutions, à des lois qui apparaissent comme la cause des limitations de jouissance et dont d'autres humains sont les représentants plus ou moins pâles. Avec le temps, le père apparaît bien davantage comme celui qui aurait vécu à une époque où l'on n'était pas soumis à de telles lois que comme celui qui s'arrogeait exclusivement la jouissance sexuelle et imposait la privation. Plusieurs opérations s'accomplissent ici en même temps. La distinction du père et des lois permet d'imaginer une époque où aucune loi n'imposait le renoncement. Parvenir à cela n'est possible qu'au prix d'une première déformation que rend possible le fait d'attribuer l'obligation du renoncement à une pleine satisfaction aux seules lois sociales. De

21. Ne nous trouvons-nous pas devant le fondement de l'interdit du toucher : ne pas toucher à certains objets pour être et ne pas être touché (battu) par le père? 
là peut être déduit qu'avant l'instauration des lois sociales aurait existé un âge d'or qui ne peut se construire que depuis l'oubli du régime de privation sexuelle que la tyrannie du père imposait à tous les fils. Dans cette reconstruction, le fils s'octroie fantasmatiquement les prérogatives qui n'appartenaient en réalité qu'au seul père. Dans cette déformation œuvre un noyau de vérité : effectivement, le père de la horde n'est ni à l'origine des interdits sociaux, ni le représentant d'une quelconque loi. Nulle loi ne le guidait, nul pacte ou contrat social ne le limitait. Son seul maître était la satisfaction directe, immédiate. Les instincts qui le mouvaient ne subissaient aucune entrave sociale. La voie du faire sans aucune pensée lui était pleinement ouverte. Mais ce que la déformation masque est ce qui est arrivé et qui a contraint, en vue de l'autoconservation, à inventer des lois limitant la jouissance de chacun et obligeant, dans la renonciation à devenir réellement le père de la horde, à composer avec les pairs.

Les interdits, les lois, le contrat ou le pacte social ne sont pas nés pour éviter que se reproduise le meurtre du père. Ils sont apparus parce que chacun des frères, avant même de ressentir la moindre culpabilité, a reculé non pas devant l'horreur du meurtre qui avait été commis, mais devant la crainte que l'audace de réaliser le désir de prendre effectivement la place du père mort n'entraîne immédiatement sa propre mise à mort. Ce n'est donc pas la morale qui est à l'origine du pacte social, ce n'est pas le sentiment de culpabilité qui est premier, mais la lâcheté. Comme le notait Freud, celle-ci fait plus souvent des hommes des hypocrites de la culture que des êtres effectivement culturels.

\section{La Vatersehnsucht s'accroît dans l'inhibition}

Devant le cadavre du père, chacun des frères est pris dans un mouvement de recul et voit naître en lui un procès mélancolique où l'acte lui paraît avoir eu lieu en vain (Freud, 1912-1913 a, p. 363). Au regard de la Vatersehnsucht, se révèle l'écart qui toujours subsistera entre le père qu'on aurait voulu être et le père que l'on pourra devenir. Depuis ce moment de vérité, s'édifie, au nom du principe de réalité, une nouvelle position de sentiment qui accompagne une modification du procès d'identification au père de la horde. Mais, ce n'est qu'au terme du processus qu'adviendra la victoire des motions de sentiment tendres du fils sur les motions hostiles qui caractérise ce nouveau positionnement des sentiments à l'égard du père. La signification de celui-ci est paradoxale, car, loin d'être un surmontement du complexe paternel, il est un acte de réparation pour l'ignominie commise sur sa personne qui se traduit à titre posthume par le triomphe suprême du père et par un accroissement, aprèscoup, de l'obéissance à son endroit. 
Ce serait nous cacher les moments préliminaires qui y conduisent que d'attribuer l'aboutissement du processus à l'action d'une culpabilité constitutionnelle. Le père mort ne deviendra pas d'emblée plus fort que ne l'avait été le père vivant. Il faut d'abord que se révèle la vanité de l'acte accompli : il satisfait certes la haine envers le père mais ne réalise pas entièrement le souhait de devenir le père. Le désir de survie a contrarié le processus d'identification en le complexifiant. Et, c'est là que survient un infléchissement du procès qui en fait un mouvement paradoxal. À la sommation d'être comme le père que subissait, au nom de son propre désir, le geste de chacun des frères vient maintenant s'ajouter celle de ne pas l'être jusqu'à l'identité complète pour éviter d'être mis à mort à son tour par les frères (Freud, 1923 b, p. 278). La formation en masse est rendue possible par le renforcement des sentiments hostiles qui, en se concentrant sur le père, prennent le dessus sur les sentiments tendres et forment le ciment qui assurera le succès de la coalition. Dans le cours de leur entreprise, chacun des frères reporte les sentiments tendres sur lui-même ou, plus exactement, sur le père auquel il s'identifie et qu'il tente de devenir effectivement dans l'accomplissement du meurtre. Le meurtre du père ne réveille pas d'emblée l'amour qu'on lui portait et la crainte qu'on en avait. Ce qui saisit le fils n'est pas l'amour pour le père qui engendrerait le remords, ce sont d'abord les sentiments tendres envers soimême, l'amour de soi et la passion de l'autoconservation qui sont renforcés devant le cadavre du père. L'amour de soi est certes à entendre dans toute sa complexité et sa duplicité, il est l'amour que «je» se porte dans le processus où il est en train de s'identifier au père. En suspendant l'accomplissement de l'identification par l'inhibition du mouvement qui conduit à se mettre effectivement à la place qu'il occupe, ce qui guide chacun des frères n'est pas l'amour pour le père dont le cadavre est à leurs pieds, mais l'amour pour le père qu'il est en train de devenir en lieu et place du père mis à mort. Si maintenant chacun réprime ses motions hostiles, c'est pour demander aux autres d'y renoncer eux-mêmes. Cette renonciation vise à sauver la possibilité de devenir plus tard le père de la horde et conduit à se contenter d'être un père qui n'a pas tous les droits de celui-là.

À partir de là, s'engage le procès qui aboutira, cette fois-ci, à la victoire, dans la création des systèmes religieux, des motions tendres sur les hostiles. L'identification au père de la horde n'ira pas jusqu'à son terme, mais son procès aura été suffisamment engagé pour permettre que l'amour pour le père que l'on était en train de devenir en lieu et place du père que l'on tuait s'étende, par sympathie, jusqu'au père exécuté. Celle-ci vient, après coup, exacerber l'amour pour le père bien au-delà de ce qu'il fut, en lui donnant une surpuissance et en contraignant à une obéissance post-mortem bien supérieure à ce 
qu'elle ne fut du vivant du père. C'est l'insuccès ${ }^{22} \mathrm{~d}$ 'un acte pourtant accompli conformément au souhait infantile qui impose le contrat social qui met en retrait la figure du père. Le retrait du père que constitue l'inhibition du processus identificatoire préserve vide la place du père mis à mort et fait entrer les fils sur la scène de l'histoire collective dont est maintenant retirée la présence incarnée du père de la horde. Ce n'est cependant qu'un renoncement provisoire car il est habité par l'attente de conditions plus propices et par l'indestructible Vatersehnsucht qui immanquablement verra sa poussée s'accroître.

L'oubli, une action décidée contre le passé

La transposition à la psychologie des masses de la formule étiologique des névroses ${ }^{23}$ a permis de penser qu'aux commencements de l'histoire culturelle de l'espèce se trouvaient des processus à contenu sexuel-agressif. Il nous est demandé d'admettre l'hypothèse d'un héritage archaïque qui seul permettrait d'entendre pleinement les conséquences durables de ces processus. La portée du procès qui est la pierre angulaire de la découverte freudienne n'est audible que depuis cette assertion audacieuse qui brouille les frontières entre l'individu et le collectif en procédant à un véritable bouleversement de leurs relations. Le Moïse confirme fermement, une fois encore, que, pour la psychanalyse, plus que le mécanisme du refoulement, c'est l'inéluctable processus du retour du refoulé, dont la force est la Vatersehnsucht, qui permet de rendre intelligible la vie psychique et le collectif. Sa reconnaissance produit un bouleversement tant de la conception du temps que de la mémoire. Le quelque chose qui est arrivé ne se « dévoile » qu'après-coup, dans son retour qui se révèle être le moment crucial où est prise ou non la décision d'oublier. L'oubli n'est en effet ni le résultat d'une passivité, ni d'une perte involontaire, il est un acte décidé contre le passé24.

Cette décision devient la caractéristique spécifique et constitutionnelle de ce point des commencements où se nouent inextricablement la survie individuelle et celle de l'espèce, et où prennent départ individuation et civilisation. Les histoires individuelles et collectives représentent l'histoire des destins de cet oubli et surtout l'histoire de son retour. Cet oubli trahit le conflit permanent qui existe entre la revendication de la pulsion et l'objection opposée par

22. Freud (1912-1913 a, p. 362), «L'insuccès est beaucoup plus favorable à la réaction morale que la satisfaction.»

23. Après cette transposition, elle s'écrit : trauma survenu dans les commencements de l'histoire culturelle humaine-défense-latence-éruption des formations religieuses sur le modèle de la formation des symptômes - retour partiel du refoulé.

24. Pour entendre la signification de ce moment décisif, voir le travail de Granoff (1975, pp. 518-549). 
la réalité. La pratique psychanalytique apprend que, face à cette alternative, l'homme ne se résout jamais à faire un choix définitif, il ne choisit ni l'un ni l'autre, ou bien plutôt il fait simultanément l'un et l'autre : il répond au conflit par deux réactions opposées, toutes deux valables et efficaces. D'une part, à l'aide du clivage, il écarte la réalité et ne se laisse rien interdire, d'autre part, dans le même souffle, il reconnaît le danger de la réalité, assume l'angoisse devant elle sous forme de symptôme et cherche ultérieurement à s'en défendre (Freud, 1940 e [1938]).

Ces deux bouleversements : celui du rapport de l'individu et du collectif, celui de la relation du temps et de la mémoire sont des éléments efficients de la troisième blessure narcissique que la psychanalyse inflige à l'homme. L'individu n'est pas une fin en soi, il est assujetti à une chaîne (Freud, $1914 c$, p. 222) qui le détermine pour le meilleur et pour le pire, et cela bien plus qu'il ne peut l'accepter ou le supporter narcissiquement. Les formations de la vie psychique et de la vie sociale portent en elles les effets d'un refoulé qui y agit de manière déterminante et qui ne peut être ramené aux seuls événements d'une vie ou de l'époque présente. Il s'agit d'un refoulé qui ne relève ni de l'expérience, ni des choix personnels mais qui fait retour sans cesse depuis la préhistoire de l'humain sous les formes les plus variées. Il révèle que des processus sexuels et agressifs originaires constituent notre actuel le plus vif, celui contre lequel ne cessent de se rebeller vainement, mais inlassablement tant l'individu que la culture qui refusent cet asservissement. Un tel positionnement a pour conséquences théoriques et techniques de forcer le psychanalyste à tempérer l'illusion d'un progrès qui conduirait vers des hauteurs toujours plus sublimes. Cela lui impose de penser, dans la vie individuelle et collective, l'œuvre quotidienne incessante de la pulsion de mort pour qui le seul véritable progrès est le retour à l'inanimé, à l'anorganique. Cela l'astreint à ne pas ignorer que l'humain fait preuve de moins d'appétit pour le changement que nous ne voudrions le croire et qu'il fait, en revanche, montre, comme le notait Proust, d'une aptitude à se plagier qui défie, bien souvent, notre entendement et l'efficience thérapeutique de la psychanalyse.

\section{L'HÉRITAGE ARCHAÏQUE}

L'hypothèse de l'héritage phylogénétique n'est pas uniquement présente dans L'Homme Moïse, elle insiste dans l'œuvre depuis Die Traumdeutung et ressurgit régulièrement tant dans les œuvres spéculatives (Actuelles sur la 
guerre et la mort, Métapsychologie, Au-delà du principe de plaisir, Le Moi et le Ça...) que dans les œuvres dites cliniques (Schreber, L'homme aux loups, Leçons d'introduction à la psychanalyse, Un enfant est battu...). En raison même de cette insistante présence, nous refuserons de la traiter comme une excroissance inutile.

Une remarque de S.J. Gould éclaire la difficulté d'aborder la question de la survivance du père, dans le psychisme, en tant qu'héritage acquis phylogénétiquement :

« Je suis conscient de traiter un problème qui, actuellement, est impopulaire. [...] J'ai fait la même très curieuse expérience plus de vingt fois : il suffit que je dise à un collègue que j'écris un livre sur les parallèles entre l'ontogenèse et la phylogenèse, pour qu'il me prenne à part, s'assure que nous sommes bien seuls, qu'il n'y a pas de micro, et me dise presque tout bas : "De vous à moi, je crois qu'en fin de compte il y a quelque chose de vrai làdedans" »(Gould, 1977, pp. 1-2, ma traduction).

Dans la construction du mode de penser analytique, deux textes occupent une place princeps : Die Traumdeutung (1900) et Totem und Tabu (1912-1914). Le premier fonde ce qu'il en est de l'élucidation psychanalytique, du point de vue individuel, l'autre du point de vue collectif. Mais nous n'ignorerons pas ce qui se joue du collectif et de la mémoire dans L'Interprétation des rêves. Après Psychologie des masses, le livre des rêves peut être lu aussi comme une analyse du moi sous le coup de la psychologie des masses dont le rêve est une illustration: une figuration des foules auxquelles nous appartenons consciemment et inconsciemment. Et nous ne négligerons pas ce que la genèse du totémisme apporte à la compréhension de la psychologie individuelle et des obstacles que la névrose de contrainte oppose au traitement psychique. Ces deux textes nous invitent à ne pas tenir la coupure entre psychologie individuelle et psychologie sociale pour plus assurée et fermement établie qu'elle ne l'est entre le pathologique et le normal. Dans un cas comme dans l'autre, l'historicité est saisie dans son rapport à des crises qui organisent l'histoire en opérant ces déplacements qui permettent sa reconstruction sans abolir l'opacité. Ces crises sont des moments où l'héritage archaïque vient à la rencontre des expériences actuelles de vie qui, elles-mêmes, s'en vont à la rencontre de l'héritage archaïque, crises où peuvent être saisis des petits bouts de véritée ${ }^{25}$. Le psychanalyste ne s'inscrit pas dans le registre du genre historiographique, il accepte que sa construction soit toujours impure, non objective. Sa méthode ne vise pas tant à dire le réel qu'à souligner avec lucidité sa présence et son action au sein de toute œuvre humaine. Il s'agit de créer une fiction plausible : un lieu où se poursuit la transposition psychique des traces mnésiques de l'événement

25. Le terme allemand Stückchen Wahrheit se trouve dans L'Homme Mö̈se. 
historique qui a influencé le cours des événements psychique. Cette méthode ne permettra certes pas de cerner complètement cet intraitable, mais elle vise un progrès de la vie de l'esprit à partir de ce qui n'a pu être effectivement effacé et qui peut être saisi partiellement, malgré les défigurations subies par ce qui est arrivé, et qui ne cesse d'arriver. La fiction porte à la fois les marques de la résistance de la chose à être saisie par les mots et celles de la résistance individuelle à accepter son retour qui s'expriment en tant qu'affects et position imaginaire. Avec la psychanalyse, la fiction fait retour dans le discours scientifique en le subvertissant : d'un côté la fiction semble certes pouvoir devenir objet du discours scientifique, mais d'un autre, la science ne peut abolir que la fiction est effectivement la forme même de son discours.

L'écriture de l'histoire par Freud se produit à partir d'événements dont « rien » ne paraît, à première vue, subsister, elle en prend, en tant que (re)construction, la place. La nécessité de poser que de tels événements ont eu historiquement lieu s'impose dès lors que l'on pense que les productions psychiques individuelles et les œuvres de la culture ne sont ni des productions ex nihilo, ni le résultat de mécanismes prédéterminés. Elles sont le produit de processus psychiques dont l'existence est postulée et qu'il faut reconstruire par induction. La reconstruction vise à expliciter logiquement une généalogie et une genèse de l'origine des mécanismes et des produits de ces processus. Elle tient lieu de l'histoire qui nous manque. Il est indiscutable que, dans la construction, rien n'est purement historique (c'est toute son ambiguïté) mais ce qui importe principalement est de rétablir un autre rapport au réel à partir de la compréhension des motifs de la perte de réalité dont se soutiennent toute organisation psychique individuelle et toute organisation culturelle. Le but de cette opération est d'entrevoir les conditions et les chemins qui pourraient atténuer cette perte. Les constructions cherchent à saisir la forme du mouvement de l'histoire, mais, nous serons toujours menacés par la tentation d'un passage réducteur de la reconstruction de ce qui aurait pu se produire à ce qui se serait produit réellement ${ }^{26}$.

\section{Transmission phylogénétique}

Sous quelle forme est présente dans la vie des peuples la tradition efficiente ou active est la question qui se pose alors. Chez l'individu, elle est réglée par l'existence dans l'inconscient des traces mnésiques du passé. S'appuyant là-dessus, Freud considère que « la concordance entre l'individu et la masse

26. Sur les concordances et divergences entre le travail de l'historien et celui du psychanalyste, voir Lévi (2006, pp. 75-98). 
est presque parfaite » et il soutient que, dans les masses aussi, l'impression du passé se conserve dans les traces mnésiques inconscientes. Un premier modèle s'appuie sur la transmission sur deux ou trois générations du souvenir conscient par les communications orales des ancêtres qui ont vécu ou été témoins des événements en question. Mais, ce modèle ne permet pas de résoudre totalement l'énigme de la transmission et la persistance temporelle efficiente de la tradition - c'est le problème de nature principielle évoqué plus haut. Sur quelques générations, la solution ne pose pas véritablement de difficulté, mais il n'en va plus ainsi quand la transmission s'accomplit sur plusieurs siècles. Le nombre de ceux qui conservent un savoir portant fidèlement témoignage se restreint avec le temps. Peut-on attribuer à ce savoir, qui n'est plus le bien de tout un peuple, mais tout au plus d'un petit cercle, " le pouvoir de s'emparer des masses avec une telle persistance quand il parvient à leur connaissance ? » (Freud, $1939 a$, p. 173). Pour Freud, cela semble peu probable et il envisage alors que « doit aussi être présent dans la masse ignorante quelque chose qui, d'une façon ou d'une autre est apparenté au savoir d'un petit nombre et qui vient à sa rencontre $»^{27}$.

Freud rappelle que, pour reconstruire et entendre quels furent les traumas précoces dont les troubles psychopathologiques sont le destin, l'expérience de la cure a montré que l'attention du psychanalyste était « attirée sur le fait vraisemblable que peuvent être à l'œuvre dans la vie psychique de l'individu non seulement des contenus vécus par lui-même, mais aussi des contenus innés, des éléments d'origine phylogénétique, un héritage archaïque » (Freud, 1939 a, p. 177). Plus loin, cette notation se voit accentuée à propos de ce qui est désigné comme les manifestations résiduelles de la cure. Certaines de ces réactions déterminées par le complexe d'Edipe et le complexe de castration ne sont pas compréhensibles en s'en tenant « strictement à ce qui a été réellement vécu par l'individu lui-même ». Elles " apparaissent injustifiées du point de vue individuel et ne deviennent compréhensibles que phylogénétiquement, du fait de leur relation à l'expérience de vie des générations antérieures » (Ibid, pp. 178-179).

Totem et Tabou pose la question des « voies et moyens dont se sert une génération pour transmettre ses états psychiques à la suivante ? » et suggère déjà que « la communication directe et la tradition [sont] loin de remplir les conditions voulues ». Freud ose, dans Moïse, son pas le plus audacieux :

«Chez l'animal-homme, au fond, il n'en irait pas non plus autrement. Aux instincts des animaux correspond son propre héritage archaïque, même s'il est d'une autre ampleur et d'un autre contenu. Après ces discussions, je n'ai aucun scrupule à énoncer que les êtres humains

27. Sur entgegenkommen : venir à la rencontre de..., voir Villa (2013). 
ont - de cette manière particulière - toujours su qu'ils ont eu autrefois un père originaire et qu'ils l'ont abattu » (Ibid., p. 180).

Et il précise :

«Une tradition qui ne se serait fondée que sur la communication ne pourrait pas engendrer le caractère de contrainte qui échoit aux phénomènes religieux. Elle serait écoutée, jugée, éventuellement écartée comme toute autre nouvelle venue de l'extérieur, elle ne parviendrait jamais au privilège de se libérer de la contrainte de la pensée logique. »

Et il conclut :

«Il faut qu'elle ait d'abord passé par le destin du refoulement, par le séjour prolongé dans l'inconscient, avant de pouvoir, lors de son retour, déployer des effets si puissants, contraindre les masses à l'emprise de la fascination, toutes choses que nous avons vues avec étonnement et jusqu'ici sans les comprendre dans la tradition religieuse »(Ibid., p. 180).

L'héritage archaïque est à entendre dans une théorie du temps où le plus archaïque s'avère être aussi actuel que les actualités du jour présent - voire plus. Loin d'être un savoir dépassé, cet héritage constitue un savoir que les hommes possèderaient toujours, d'une manière inconsciente. Il détermine non seulement leur être au monde mais aussi, selon les formes d'acceptation ou de refus de cet héritage, les formes d'organisation sociale et les modalités des systèmes de croyance qui les sous-tendent.

\section{Les caractéristiques de l'héritage archaïque}

Pour Freud, le ça serait porteur héréditairement des traces de certaines expériences vécues par les « moi » de nos ancêtres (Freud, 1923 b, p. 282), celles-ci seraient équivalentes aux traces laissées par des expériences vécues personnellement : elles auraient la même actualité. C'est la répétition fréquente et/ou la forte intensité de ces expériences chez de nombreux ancêtres qui seraient la condition de la transposition des traces d'expériences vécues par les ancêtres en traces héritables par tous les descendants de l'espèce: les expériences vécues du moi se transposent en expériences vécues du ça (Ibid. $)^{28}$. Cette proposition nous invite à penser la coexistence de diverses sortes de moi doté de «propriétés héritées et de propriétés acquises» que nous ne sommes pas à même de distinguer entre elles. À l'objection qu' « aucun moi n'est présent au début de la vie individuelle », il répond que nous ne devons pas « oublier qu'à l'origine ça et moi sont un » et que, par conséquent, le moi, dès sa première différenciation d'avec le ça, est porteur, déterminé, informé par l'héritage

28. Ibid et Freud (1937 c, p. 43). 
archä̈que. L'effet de ce dernier «consiste en des dispositions déterminées, telles qu'elles sont propres à tous les êtres vivants. Donc en l'aptitude et le penchant à emprunter des orientations de développement déterminées et à réagir d'une manière particulière à certaines excitations, impressions et stimuli » (Freud, $1939 a$, p. 177, p. 179). Cet héritage viendrait dans l'espèce humaine au lieu qu'occupent les instincts chez les autres animaux. Chaque enfant, en venant au monde, le porterait en lui comme témoignage de l'influence que les expériences vécues par ses ancêtres exercent, bien avant toute expérience propre (Freud, $1940 a$ [1938], p. 257), sur son devenir, en tant que « schème phylogénétique qui s'accomplit ${ }^{29} »$.

Freud considère dans la XIII ${ }^{\mathrm{e}}$ des Leçons d'introduction à la psychanalyse que :

« le point de vue phylogénétique est dissimulé en partie chez l'être humain du fait que ce qui est au fond transmis héréditairement n'en est pas moins acquis à nouveau dans le développement individuel, vraisemblablement parce que persistent encore et agissent sur chaque individu les mêmes circonstances qui ont en leur temps nécessité cette acquisition. Je voudrais dire qu'en leur temps leur effet a été de créer et qu'il est maintenant de faire revenir » (Freud, 1916-1917 $a$ [1915-1917]).

Cette « exagération » freudienne nous confronte à la question de la temporalité mise en œuvre dans cette transposition, elle n'est pas immédiate, elle ne se ferait pas spontanément à la suite d'une unique survenue de l'expérience. Pour que l'expérience soit transposable, elle doit se répéter avec une certaine fréquence chez un certain nombre d'individus et posséder une certaine force d'impact. Mais, dans Moïse, Freud n'exclut pas qu'un événement unique suffisamment important puisse aussi posséder ce pouvoir de transposition.

\section{Le ça est-il modifiable?}

Nous nous tournons vers le travail de Nathalie Zaltzman ${ }^{30}$ et son hypothèse qu'il faut envisager un espace-temps mixte et hybride entre le temps ontogénétique et le temps phylogénétique. C'est pendant ce temps que se déroulerait ce travail de transposition d'une génération à une autre qui a pour préalable le travail de transposition individuelle de l'expérience vécue du moi en expérience du ça. La distinction entre les expériences vécues du moi et les expériences du ça nous indique que toute expérience vécue par le moi n'est pas automatiquement et toujours une expérience vécue par le ça. Ne doit-on pas envisager

29. Freud (1918 b, p. 84): « Sur ce point l'hérédité triomphe de l'expérience de vie accidentelle. »

30. Ce qui suit est une reprise remaniée d'un travail fait avec Eva Weil (2011). 
deux cas de figure ? Dans le premier cas, il existerait des expériences du moi qui n'auraient aucune conséquence sur le ça, elles n'y laisseraient pas de traces spécifiques et elles ne le modifieraient pas. Dans le second cas de figure, nous aurions à faire à des expériences du moi qui affectent le ça en s'y transposant. Ce qui serait arrivé au moi le toucherait jusqu'au point de franchir ses limites, de s'étendre jusqu'au ça en exigeant un travail spécifique de transposition. C'est la notion de latence (Weil, 2000, pp. 169-179) qui rendrait compte de ce temps où l'expérience du moi n'est pas encore expérience du ça et où pourrait survenir une modification du ça qui serait héréditaire.

La transposition d'une expérience du moi en expérience du ça n'est pas immédiate. Elle ne se fait pas spontanément. Nous retrouvons, ici, toute la problématique du statut de l'actuel dans l'Interprétation des rêves avec en son centre l'effet de l'après-coup qui permet le passage de la trace de la perception à l'état de trace mnésique. "Ce qui est arrivé » serait mis en latence tant que sa «fréquence » ou son «intensité » n'auraient pas été assez marquantes pour contraindre à la transposition. Cela serait arrivé « sans avoir lieu psychique » : des traces sans représentations qui n'ont pas trouvé leurs mots et depuis lesquelles aucun récit audible collectivement et individuellement ne peut se déployer. Au-delà d'une certaine intensité et d'une certaine fréquence, se produirait une fracture des limites du moi dont les contenus se déverseraient dans le ça qui en serait modifié. La question se pose alors de savoir comment ce ça modifié fait retour vers le moi qui a ou n'a pas reconstruit ses limites. La latence ne serait-elle pas aussi ce temps nécessaire au moi pour admettre les effets et conséquences de la modification du ça ? L'expérience que représente pour le moi les poussées du ça modifié n'est pas admissible d'emblée, elle impose un temps de connaissance et de reconnaissance.

$\mathrm{Ne}$ faut-il pas éviter l'impasse de resituer la question uniquement au niveau du sujet individuel ? La reconnaissance individuelle est indissolublement dépendante de la reconnaissance collective et déterminée par elle (Weil, 2009). Le temps de latence ne permettrait-il pas la mise en résonance et en concordance entre le collectif et une expérience vécue non réductible à une affaire uniquement personnelle ? Lorsque le collectif reconnaît que le vécu du sujet relève de la commune appartenance à l'espèce et à son histoire, l'expérience, sans trouver complètement sens, cesse d'appartenir à du pur non-sens. Qu'est-ce qui rend possible l'émergence de cet écho ? Quel espace de temps est-il nécessaire ? Trente, quarante ans semblent en être la mesure.

Il y aurait latence parce que les moyens de représenter ne sont pas là, mais il ne s'agit pas seulement des moyens individuels, mais des moyens résultant d'une inscription de l'individu dans le collectif et du collectif dans l'individu. L'absence de moyens aurait pour effet de faire que l'homme se sente seul et 
sans autre humain, qu'il éprouve une crainte de l'effondrement, une peur de tomber hors du monde, un sentiment de désolation et qu'il estime que ne pourraient le comprendre que ceux qui ont vécus la même chose - et encore.

\section{LA CONTRAINTE D’UNE HYPOTHÈSE CONTROVERSÉE}

Achever L'Homme Moïse et le publier forment un acte que Freud se sentait impérieusement tenu d'accomplir avant de mourir. Avec ce livre, il nous demande, non sans quelques scrupules, d'accuser réception d'un ensemble d'hypothèses dont la teneur risque d'entacher la scientificité de la démarche et de la théorie psychanalytiques - caractère auquel il tenait fortement. C'est une nécessité vitale qui enjoint à Freud l'audace de publier dans un ouvrage déjà peu orthodoxe, une hypothèse biologique allant à l'encontre de « la position présente de la science biologique » (Freud, 1939 a, p. 179). Prendre ce risque lui est imposé par cette période où se joue la survie du monde et donc aussi de la psychanalyse.

Cette position ne saurait être réduite à ce qu'on appelle trop hâtivement le psycho-lamarckisme de Freud. La biologie de l'époque, par la voix de l'un de ses représentants les plus éminents : August Weismann, rejette la possibilité de la transmission héréditaire aux descendants des particularités acquises au cours d'une vie. Mais, pour Freud, si l'on essaie de comprendre comment les progrès de la vie de l'esprit, qui s'expriment par des acquis culturels, des traditions, des traits propres à un peuple ou à une nation, se transmettent de génération en génération, on ne peut pas se contenter d'une explication par la transmission transgénérationnelle, par le langage, par le bain culturel ou d'inconscient à inconscient. Ces modes de transmission ne rendent pas compte de la consistance d'une transmission qui semble défier le temps et dont les éléments ne présentent pas la fragilité que possèdent les acquisitions liées à l'éducation et à l'influence des générations contemporaines. Le recours à une autre explication devient logique. Ce qui est transmis semble inhérent au sujet en tant que prédispositions qui n'attendent qu'une occasion propice pour se déployer. Elles se développeront, certes, plus aisément si l'éducation, allant dans le sens des lignes tracées par la constitution, les renforce en leur imprimant une forme plus nette et plus profonde (Freud, $1905 d$, pp. 191-192). De ce point de vue, on peut se demander ce qui se passe quand l'éducation ne va pas dans le même sens que les lignes prédéterminées et/ou qu'elle s'y oppose ou les contrecarre. Pour Freud, la réponse est claire, à savoir qu'à la suite d'un certain vécu plus récent peut survenir une modification de la constitution 
de l'individu. La question de savoir si cette modification survenue à l'échelle individuelle serait sous certaines conditions transmissible aux générations suivantes, ressurgit alors.

Freud nous dit donc quelque chose comme : "Je sais bien que les faits biologiques semblent invalider l'hypothèse à laquelle je recours, mais je suis contraint inévitablement à cette audace car je rencontre des faits psychiques, tout aussi réels et consistants, qui insistent et persistent comme des manifestations résiduelles du travail psychanalytique dont nous ne parviendrons pas à rendre compte théoriquement sans le recours à une hypothèse de ce type ».

Freud savait donc parfaitement que la biologie prétendait avoir démontré que la transmission des caractères acquis est impossible. Pourtant, comme Ernest Jones le signale avec quelque irritation :

« Freud ne renonça jamais à un iota de sa conviction dans la transmission héréditaire de caractères acquis. Je découvris à quel point il était inébranlable sur la question lorsque, pendant la dernière année de sa vie, je discutais avec lui d'une phrase de son livre sur Moïse dans laquelle il exprimait le point de vue lamarckien en termes universels et que je désirais lui voir modifier. Je lui dis qu'il avait le droit, bien sûr, de soutenir n'importe quelle opinion dans sa propre spécialité, la psychologie, même si elle allait à l'encontre de tous les principes biologiques, mais je le suppliais d'omettre le passage dans lequel il l'appliquait à tout le domaine de l'évolution biologique, étant donné qu'aucun biologiste responsable ne la considérait comme actuellement défendable. En réponse, il ne put que me dire qu'ils avaient tous tort et qu'il ne supprimerait pas le passage en question. [...] Il n'est pas facile d'expliquer la rigidité avec laquelle Freud s'en tint à cette opinion et la détermination avec laquelle il ignora tous les arguments biologiques prouvant le contraire »(Jones, 2006, p. 356).

S'agit-il vraiment de rigidité, comme le pense Jones, ou d'une rigueur méthodologique, d'une exigence épistémologique devant laquelle Freud ne se dérobe pas et qui le rend incurable de cette hypothèse qu'il faudrait selon Jones et Huxley (1953, p. 40) $)^{31}$ ranger parmi les vieilles superstitions?

Toujours est-il qu'après avoir été amplement brocardée et stigmatisée comme l'un des supposés fourvoiements biologiques de Freud, cette incurable croyance freudienne a retrouvé sa dignité d'hypothèse scientifique et mérite donc qu'on la considère enfin avec plus de respect qu'il ne lui en fut témoigné. Il est vrai que pendant un certain temps, l'impossibilité de l'hérédité de l'acquis était posée comme une vérité définitive. Selon la doxa, seules étaient transmissibles les modifications du génome ; les modifications acquises du phénotype ne caractérisaient qu'un individu, elles n'agissaient pas sur le génome et n'étaient donc pas transmissibles. Les recherches conduites dans le domaine de l'épigenèse, depuis les années 1940 (peu de temps après la

31. Je dois mes connaissances sur l'épigénétique aux échanges avec le Pr. Jonathan Weitzman (UMR Epigenetics and Cell Fate, CNRs/Université Paris-Diderot) et avec la Pr. Eva Jablonka (Cohn Institute for the History and Philosophy of Sciences and Ideas, Tel-Aviv University), Jablonka (2005; 2011). 


\section{François Villa}

publication du Moïse) et plus particulièrement dans les vingt dernières années, sont en train de redistribuer les cartes du savoir. Il est maintenant établi que, dans le monde animal, certaines expériences vécues par un individu produisent des modifications du génome qui vont être retrouvées chez ses descendants. L'épigénome est porteur d'une hérédité qui est fondamentale au niveau cellulaire car elle contribue à doter les cellules d'une «mémoire » de leur « identité ». La sélection naturelle ne porte plus directement sur les gènes, mais sur la capacité des organismes à réagir de telle ou telle façon aux problèmes d'adaptation que leur pose le milieu. Cette faculté n'implique pas seulement le système des gènes déjà acquis. Les expériences de Waddington ${ }^{32}$ montrent que le même caractère peut être produit soit par une modification environnementale, soit par une modification génétique. De cette façon, les dispositions, les aptitudes et les penchants acquis sont héritables et constituent des prédéterminations à réagir d'une manière particulière à certaines excitations, impressions et stimuli qui ressemblent à ceux de l'expérience vécue par les ascendants ${ }^{33}$.

Nous faisons référence à ces recherches pour indiquer qu'il n'est plus aussi aisé que par le passé d'exclure l'exagération freudienne. Celle-ci retrouve une certaine acuité et pourrait donc comporter un noyau de vérité. Mais, nous tenons à souligner que, même sans l'évocation des recherches en épigénétique, nous n'aurions pas pu nous débarrasser d'un revers de main de cette hypothèse en nous dispensant de saisir la nécessité logique qui contraignit Freud à la maintenir. L'hypothèse de l'héritage archaïque exige une réorganisation de nos savoirs antérieurs, un véritable remaniement de nos modes de pensée et il engendre un réel malaise. Peut-être est-ce l'une des raisons qui fait du Moïse un livre toujours dérangeant.

\section{LES FINS DE LA PSYCHANALYSE}

Tenir ensemble phylogenèse et ontogenèse

La place accordée dans la réélaboration de la théorie traumatique à l'événement de réalité dans l'origine de la névrose peut déconcerter en donnant le sentiment que la première théorie traumatique reprend avec vigueur cette

32. Conrad Waddington introduit le terme d'épigénétique en 1942.

33. Bien d'autres recherches dont les résultats confirment l'hypothèse de Waddington ont été faites depuis, voir Matthews, Phillips (2010). La brève mention de ces recherches épigénétiques fait entrevoir des pistes de recherches tant à l'intérieur de la psychanalyse que dans l'échange avec les épigénéticiens. C'est l'un des objets du Labex Who Am I ? Déterminants de l'identité, de la molécule à l'individu (Université Paris Diderot) coordonné par Jonathan Weitzman et Catherine Dargemont et dont je suis l'un des promoteurs. 
interrogation lancinante sur la réalité et la vérité historique. Le lecteur de L'Homme aux loups ressent un sentiment similaire lorsque Freud revient de manière insistante sur la question de savoir si le patient a été le témoin d'un coït parental ou s'il s'agit d'une construction fantasmatique. Dans les deux cas, Freud prétend d'ailleurs se sortir de cet embarras avec la même solution : l'événement traumatique ne relèverait pas nécessairement de l'histoire individuelle, mais appartiendrait à celle de l'espèce et ses traces seraient présentes, dès les commencements, en chaque individu comme héritage archaïque.

Dans les deux contextes, il ne serait pas illégitime de penser que Freud nous invite au jeu du qui perd gagne: soit l'analyste trouve dans l'histoire de vie du patient, les événements accidentels qui donnent accès à la compréhension de son état psychique, soit, lorsque de tels événements semblent absents de cette histoire, il recourt à l'explication phylogénétique en invoquant des événements préhistorique effectifs. Mais, n'y a-t-il pas, plutôt, en ce point, un enjeu épistémologique majeur qui détermine la vision théorique que le psychanalyste peut avoir des fins de la psychanalyse et dont découlent des conséquences techniques essentielles pour la méthode psychanalytique ? Les textes contemporains du Moïse confortent cette opinion en explorant, du point de vue du traitement psychique, ces conséquences.

Il n'y a de fait pas d'alternative. Le seul parti qui nous est proposé est non seulement de penser ensemble ces deux dimensions, mais d'envisager, qu'en particulier dans l'espèce humaine, l'ontogenèse est la condition de l'accomplissement phylogénétique. L'ontogenèse est le processus qui résulte des interprétations de son héritage qu'un individu peut faire à la faveur des contingences du quotidien. Le traitement psychique de notre surdétermination par le constitutionnel et par l'accidentel fait advenir cette histoire singulière.

Le développement de la méthode et du mode de penser psychanalytiques n'a été possible, comme nous le savons, qu'à partir de la réévaluation, imposée par l'expérience du traitement psychique, de la part prise par l'accidentel dans la formation de la névrose. Cette réévaluation ne conduisit jamais à revenir sur la découverte dans le traitement hypnotique du rôle essentiel de ce facteur dans la survenue non seulement d'une névrose, mais de toutes les activités psychiques. Mais elle aboutit à deux conséquences. La première fut de se rendre compte que, porté par les succès thérapeutiques permis par la prise en considération du facteur accidentel, son influence avait été surestimée - au point de lui imputer, dans un premier temps, le rôle principal dans l'éclosion de la névrose. L'autre conséquence fut, contrepoint logique, la sous-estimation du facteur constitutionnel et héréditaire. L'expérience clinique montrera que les séductions sexuelles précoces, présentes indéniablement dans nombre de 
cures, ne suffisent pas à expliciter le processus névrotique. Ce fait conduira à former l'hypothèse qu'il y a une fragilité constitutionnelle de la «fonction » sexuelle qui prédispose l'humain à la névrose et qui n'attend que l'occasion de s'exprimer en prenant la forme de l'un de ses destins psychiques possible (Freud, $1898 a ; 1906 a$ [1905]).

Dans le cours des transformations, des complexifications que connaîtra la théorie des névroses, la valeur accordée à la sexualité, à l'infantilisme de l'appareil sexué et de l'instrument de l'âme, à l'effet d'après-coup ne sera jamais, en revanche, ni contesté, ni renié (Freud, 1898 a, p. 236). Le Moïse est, à la fois, un témoignage de la persistance de ces points de vue et une reconsidération de leur valeur et de leur articulation. Mais, le recours à l'hypothèse phylogénétique ne comporterait-il pas la menace de détourner la psychanalyse de ce qui serait le cœur de sa pratique (la vie singulière du patient, sa réalité psychique propre) vers des généralités, vers des interprétations universalistes et symbolistes, vers une interprétation anagogique, vers une conception mystique du monde? L'idéal méthodologique, pour Freud, serait de penser la clinique depuis ces deux points de vue que sont la phylogenèse et l'ontogenèse en ne perdant jamais de vue que le processus d'individuation n'advient que des effets d'après-coup qui surviennent de la conjonction du constitutionnel et de l'accidentel. Sans le secours des hasards ${ }^{34}$ de la vie, la constitution n'aurait pas le pouvoir d'enclencher le processus.

Phylogenèse et ontogenèse : effet paradoxal de dissimulation réciproque

Tenir ensemble ces deux dimensions ne semble être possible qu'incidemment et seulement pendant de brefs et rares instants. Notre pente naturelle est d'opérer une disjonction exclusive entre ces perspectives, ce qui produit, presque toujours, un effet paradoxal de dissimulation d'un facteur par l'autre. Lorsque l'accent est mis sur l'un des deux, la conséquence en est presque toujours non pas simplement la sous-estimation de l'autre, mais le quasi effacement de sa prise en considération. C'est là sans doute l'expression d'une résistance narcissique à accepter que nous ne sommes ni au principe de notre vie, ni une fin en soi, mais un des maillons d'une chaîne qui a commencé bien avant notre venue au monde et qui perdurera au-delà de notre mort. L'humain semble avoir une forte propension à négliger le poids du phylogénétique et du hasard dans son développement psychique et dans la constitution de ce qu'il reconnaît comme son identité. Si chacun reste indéniablement l'interprète

34. Le premier hasard étant la rencontre bien opportune du point de vue psychique de l'aide étrangère. 
singulier de cette surdétermination par la constitution et par la contingence, il ne peut, semble-t-il, qu'ignorer structurellement que la forme prise par notre vie est déterminée tout autant par les expériences de vie accidentelles que par les héritages dont nous sommes dépositaires à notre insu. Cette ignorance structurelle serait renforcée par les fantasmes d'auto-engendrement et d'autosuffisance.

$\mathrm{Au}$ jeu de la dissimulation réciproque, le point de vue phylogénétique est celui qui est le plus fréquemment escamoté. Il est même possible que la pratique quotidienne du psychanalyste ait, à l'encontre de ce qui devrait être sa visée, pour effet de renforcer ce handicap. Que, dans la cure, on puisse en venir à négliger le point de vue phylogénétique est assez facilement compréhensible. Comme nous l'avons rappelé la méthode psychanalytique naît de la prise de conscience du rôle de l'accidentel dans la vie psychique. Même après la réévaluation de sa fonction étiologique, il demeurera vrai, comme le réaffirme L'Analyse finie et l'Analyse infinie, que c'est dans les cas où le facteur accidentel est prépondérant dans la série complémentaire que l'analyse réalisera au mieux ce dont elle est capable en permettant de « remplacer, grâce au renforcement du moi, une décision inadéquate remontant aux tout premiers temps par une liquidation correcte » (Freud, 1937 c, pp. 21-22).

Il est certain que l'application correcte de la méthode dans le dispositif analytique instaure une situation d'accentuation de l'attention portée aux productions incidentes où s'éprouve effectivement l'existence de la réalité psychique ; cela favorise indéniablement la reconnaissance de la part prise par les contingences dans ce que nous devenons. En un mot, la psychanalyse voit venir au premier plan ce sur quoi elle peut agir et qui peut être réellement modifié par le traitement psychique. "L'accidentel joue en effet le rôle principal dans l'analyse [...] les facteurs accidentels sont mis en avant, les facteurs dispositionnels laissés à l'arrière-plan et le développement ontogénétique est pris en compte avant le développement phylogénétique ; il est maîtrisé par elle presque sans reste ${ }^{35}$. »

Défense et illustration du rôle de l'accidentel et de l'héritage

$\mathrm{Au}$ point où nous nous trouvons, le lecteur pourrait légitimement nous interpeller : «à vous suivre, le constitutionnel, l'héritage archaïque serait toujours dissimulé, il ne serait jamais observable positivement, de manière manifeste. Certes, vous affirmez qu'il est toujours présent et agissant à l'arrière-plan 
comme ce fond d'où émergeraient, grâce au concours de l'accidentel, toutes les formes de vie psychique individuelle et collective. Mais sur quoi de concret, d'indiscutable se fonde cette affirmation, cette spéculation? Ne s'agirait-il pas simplement d'une croyance que vous partageriez avec Freud et à laquelle vous souhaiteriez que nous adhérions aveuglement ? Ne devriez-vous pas nous fournir une vignette, un exemple, une situation clinique qui viendrait étayer votre position? »

Nous entendons le bien fondé de cette demande, mais nous devons concéder que nous ne sommes pas en mesure de la satisfaire en fournissant cette preuve par la clinique qui emporterait la décision. Autant il nous serait possible de présenter, et nous l'avons fait plus d'une fois, des moments de cure qui constitueraient une défense et illustration soit du rôle joué par l'accidentel, soit de la transmission de «secrets de famille », d'événements cachés, occultés ou refoulés sur quelques générations (deux, trois, voire un peu plus), autant il nous semble impossible de le faire de manière positive pour la transmission de l'héritage archaïque. Celui-ci œuvre toujours au négatif.

La nécessité de postuler son existence est induite dans une cure psychanalytique par la rencontre de ces lacunes ou de ces doutes sur l'ordonnancement temporel ou logique des faits psychiques qui subsistent alors même que le travail interprétatif a permis un réel processus de remémoration. Ces lacunes, pour être surmontées, convoquent la nécessité d'un travail de construction. Nous conviendrons que le recours à l'hypothèse phylogénétique dans L'Homme aux loups pour tenter de surmonter les énigmes rencontrées dans cure n'apporte pas de preuve définitive en faveur du postulat. Le phylogénétique y est d'abord convoqué pour comprendre pourquoi c'est le père qui devient ce personnage effrayant de qui provient la menace de la castration alors même que, dans la réalité de l'expérience vécue, les menaces procédaient plutôt des femmes. La solution proposée pour sortir de ce dilemme est que le patient ne ferait qu'accomplir un schème phylogénétique qui a une force telle qu'il triomphe de l'expérience de vie accidentelle. Le second recours survient lorsque Freud veut savoir si chez son patient la scène originaire était fantaisie ou expérience vécue réelle. Bien qu'il penche fortement pour le second terme de l'alternative, un doute subsiste. Il propose de le dépasser en soutenant que, dans l'histoire originaire de la névrose, là où la propre expérience de vie ne suffit pas, le petit humain « comble les lacunes de la vérité individuelle avec de la vérité préhistorique, introduit l'expérience des ancêtres à la place de l'expérience propre ». Ces deux moments de cure engagent la question du rapport au père : c'est de celui qui possède sexuellement la mère dans la scène originaire que provient la menace de castration. 
Les lecteurs sceptiques rappelleront que ce texte ne fait appel à cette hypothèse que dans une argumentation contre Jung. Le but n'en serait pas tant de défendre le phylogénétique que d'appeler son disciple à la prudence quant à l'usage à faire dans la cure des sources religieuses et mythiques. Freud remet en cause l'usage interprétatif que Jung fait des mythes en les appliquant comme une réalité en soi qui interviendrait dans toute cure dans une quasi totale indépendance des particularités de l'histoire individuelle. Le recours à ces sources procure, selon Freud, l'avantage douteux de pouvoir se débarrasser de tout le choquant dont est porteur le complexe parental avec la place qu'y tient la chose sexuelle. La différence entre les deux approches réside dans le fondement ontologique de la pensée symbolique ${ }^{36}$. À l'encontre de la démarche jungienne, pour Freud, les thèmes mythiques et religieux procèdent d'un procès historique qui n'est pas réductible à la seule histoire individuelle, mais relève fondamentalement de celle de l'espèce. L'héritage archaïque ne serait pas constitué par les contenus des mythes en tant que tel. Il formerait un fonds impersonnel qualifié d'instinctuel qui serait "quelque chose comme une préparation à comprendre » (Freud, 1918 b, pp. 116-117). Celui-ci exercerait dès les commencements une action sur l'humain en prédéterminant son inscription dans la tradition de ses proches. Ce fonds est le noyau de l'inconscient produit lors d'un refoulement organique originaire dont le but était de surmonter l'infantilisme sexuel. En lui, subsistent agissantes l'ambivalence à l'égard du père, les traces du forfait commis et de la Vatersehnsucht exacerbée par l'insuccès de l'acte meurtrier. Ce sont les sources phylogénétiques de l'apparition et du développement du phénomène religieux qui persistent encore et agissent sur chaque individu. Dès que se reproduisent des circonstances similaires à la situation originaire où est apparue la nécessité du religieux, elles reviennent sur le devant de la scène dans un processus du retour du refoulé, en créant les conditions non seulement de la réacquisition de l'héritage par sa recréation, mais aussi de sa possible modification. Pour la psychanalyse freudienne, l'universalité du processus et des formes de création mythique, l'universalité de la symbolique du langage ne peuvent s'entendre qu'à partir de la communauté originaire d'affect que reçoivent en héritage les hommes et dont ils ont à faire une communauté de destin.

La différence des approches freudiennes et jungiennes nous importe en raison des conséquences méthodologiques et techniques qu'elle a pour le travail quotidien du psychanalyste. Dans la tentation d'une modalité d'interprétation symboliste, proche d'un modèle clef des songes, Freud ne voit que des menaces d'altération et d'atténuation de la radicalité de la technique et de la

36. Pour le débat avec Jung, voir Widlöcher (1966). 
théorie psychanalytique. En émoussant le rôle du facteur sexuel et du complexe paternel dans la vie psychique, il est vraisemblable que nombre de résistances à la psychanalyse tomberont. Mais le prix en est lourd. Un premier risque est le détournement vers une vision du monde non scientifique, tentée par le mysticisme. Le second est de se tourner vers une pratique qui, restant soumise au principe de plaisir, serait déterminée par des préoccupations éducatives, par un souci de réconciliation du sujet avec des pseudo valeurs esthétiques et morales éternelles, par des visées intégratives, qui respecteraient la passion synthétique du moi. Il y aurait un abandon de l'amour matérialiste de la vérité qui est au fondement de l'entreprise analytique et qui en constitue une des fins.

La position psychanalytique se révèle intransigeante. L'évolution individuelle est bien plus que ne le conçoit le moi organiquement déterminée et héréditairement fixée au point de pouvoir même à l'occasion s'effectuer sans le moindre concours de l'éducation (Freud S., $1905 d$, p. 113), mais la première tâche de la psychanalyse reste de découvrir et de suivre comment, en tirant parti des coups du sort, a été mis singulièrement en acte ce programme en puissance. Tout au long de l'œuvre freudienne sera réaffirmé fermement qu'avant d'avoir respecté la séquence d'instances correctes, il n'est pas admissible de convoquer à des fins interprétatives le rôle joué par le facteur héréditaire acquis par phylogenèse dans la vie d'âme ${ }^{37}$. Avant d'en arriver là, nous devons avoir déployé dans la cure tous les moyens techniques dont nous disposons pour expliquer l'état psychique du patient à partir des événements psychiques de l'histoire individuelle.

En quoi la psychanalyse a-t-elle besoin de l'hypothèse phylogénétique?

L'efficacité de la méthode psychanalytique consiste dans la redécouverte, au cours de la cure, par le patient de la part qu'il a prise dans le destin donné à sa vie. L'expérience psychanalytique, en repassant par tous les méandres d'une vie, permet la prise de conscience par le patient de sa part de responsabilité dans ce qu'il a vécu et dans ce qu'il vit aujourd'hui. Elle permet de s'arracher à l'idée que la vie ne serait que le résultat d'une fatalité inexorable et aveugle où il n'aurait fait que subir passivement la conséquence d'événements, de circonstances et de comportements dont il n'aurait été que la victime. Le travail de la remémoration permet de percevoir à nouveau ces croisées de chemins qui ont été des moments où l'état pulsionnel du sujet a été confronté à l'état

37. Nunberg, Federn (1976), Les premiers psychanalystes. Minutes de la Société psychanalytique de Vienne, III, (8 novembre 1911). Freud (1918 b, p. 117). 
réel du monde. Il a alors prononcé un jugement sur cette réalité. Tirant les conséquences de celui-ci, il s'est décidé, en s'étayant sur les contingences, à prendre un parti qui a infléchi le cours de sa vie vers la forme qu'elle a aujourd'hui. Cette dernière n'est que la figure de l'un des destins possibles qui s'offraient à ce carrefour de la vie. L'expérience de la cure montre qu'il est possible d'opérer aujourd'hui soit le même choix, soit d'autres choix. Et il s'avère que, quel que soit alors le choix fait, dans la mesure où il se fait dans une plus grande connaissance de ses causes, il s'ensuit une modification ${ }^{38} \mathrm{du}$ regard que le patient porte sur lui-même, sa vie et les autres ainsi que de ses modes de vivre et d'exister. Les modifications permises par la psychanalyse portent sur ce qui est mais peut tout aussi bien être autrement, c'est-à-dire sur les figures du destin différent qui ont pu se construire depuis le fonds héréditaire commun. Sur celui-ci, elle n'a, a priori, aucune action correctrice possible, il constitue un socle qui est donné par l'héritage qui est ce qui est et ne peut pas être autrement. Nous avons dit a priori, car, fidèle à l'évolutionnisme, Freud est loin d'exclure la possibilité que, sous certaines conditions, certains événements aient une intensité qui entraîne une modification de l'héritage transmissible. Nous partageons ce point de vue.

Notre travail quotidien nous impose d'être pris dans les plis et replis de la réalité psychique individuelle pour en entendre, dans le in vivo du transfert, les déterminants, les motifs, les visées et les effets. Parvenir à une juste évaluation du pouvoir de la parole et de son champ d'efficacité n'est pas l'un des moindres enjeux de la cure et de l'appréciation de son succès ou de son échec. Le dispositif analytique, à l'encontre des exigences civilisatrices qui l'atténuent, accentue par nécessité méthodologique le pouvoir magique des mots et redonne, pendant une bonne partie de la cure, une vigueur à la croyance en la toute-puissance de la pensée. Prendre conscience du rôle joué par cette croyance et y renoncer constituent, dans notre expérience, l'un des points névralgiques où se joue le devenir culturel de la cure. En ce point s'ouvre la possibilité de sa terminaison et se " retrouve », par accroissement de la capacité d'aimer et de travailler, la force d'affronter courageusement et avec détermination le réel. Le psychanalyste, pour exercer son art, se voit contraint d'épouser réellement cette croyance en la toute-puissance mais il doit se méfier de ne pas y adhérer en succombant à la propension à y croire aveuglement qui est commune à tous les hommes. Lorsqu'il cède à cette tentation, il peut, d'une part, devenir inapte à penser que la psychanalyse ne peut que rencontrer immanquablement des limites au-delà desquelles cesse son efficacité et, d'autre part, croire en une

38. Rien ne garantit que la modification sera nécessairement bénéfique. 
toute-puissance de la psychanalyse qui pourrait contribuer à rendre la cure interminable.

En paraphrasant Freud (1900 a,p. 146 note 1, p. 578), je dirai que, même dans les cas les mieux analysés, est rencontré toujours un point qui non seulement reste dans l'obscurité, mais dont on ressent qu'on doit (impératif catégorique) le laisser ainsi. Il faut accepter que quelque chose reste sans achèvement et cela alors même que s'impose l'évidence que tout le réseau des associations converge vers cet ombilic où se fait entendre, au-delà de tous les énoncés, la force agissante du souhait infantile. Nous pensons qu'en ce point, l'histoire singulière touche au non-connu de l'héritage archaïque dont elle prend son essor et dont la reconnaissance de l'existence est un accroissement de connaissance.

Les limites de l'art psychanalytique

Mais, mon objecteur revient à la charge : " J'entends qu'il est important techniquement et méthodologiquement pour la conduite de la cure d'envisager et de reconnaître les limites de son action, je vois bien les dangers qui, pour le travail du psychanalyste, résulteraient de la méconnaissance de celles-ci, mais pour éviter cette menace a-t-on vraiment besoin de faire appel au phylogénétique ? Ne suffit-il pas d'attirer l'attention de l'analyste sur les risques que ferait encourir la croyance en la toute-puissance de l'esprit? Ne peut-on pas penser que sa propre expérience d'analysant et la formation analytique qu'il a suivie lui ont appris à reconnaître et les limites de sa propre psychanalyse, et les limites personnelles qu'elle lui a révélées ? Et, si cela ne suffisait pas, croyez-vous vraiment que l'explication par le fonds héréditaire est la seule à laquelle nous puissions faire appel ?»

J'accuse réception d'une telle objection en reconnaissant que je ne dispose toujours pas de preuve plus probante. Mais, retenant que mon interlocuteur a été sensible à la question de la reconnaissance des limites de notre pratique, je lui dirai que mon expérience de psychanalysant, de psychanalyste et de la communauté des psychanalystes me laisse dubitatif sur la garantie contre le fantasme de toute-puissance que constitueraient cure et formation personnelles. Et, même si celles-ci créaient un garde-fou suffisant, cela ne nous apprendrait rien sur la nature de ce point ombilical et je maintiendrai que quelque chose d'analogique à l'héritage archaïque reste à concevoir, à construire.

La clinique ne nous donne pas les moyens de prouver positivement la pertinence de l'hypothèse phylogénétique. Elle demeure cependant le lieu où se fait ressentir sa nécessité ou, pour le moins, l'exigence d'une 
construction théorique rendant compte d'une manière suffisante de certaines difficultés qui se retrouvent inéluctablement et répétitivement dans la plupart des cures, pour ne pas dire dans toutes. Car, plus que l'exemple d'une cure singulière, c'est la série que constitue l'ensemble des cures conduites par le psychanalyste dans la longue durée qui nous pousse vers l'hypothèse $\mathrm{du}$ fonds constitutionnel commun. Avec le temps, s'impose dans la pratique le sentiment que, malgré la diversité et la richesse des méandres de la petite histoire, il y a bien quelque chose de commun à toutes ces histoires personnelles, comme un point d'invariance culturelle universel. Il y a un air de famille qui unit les humains au-delà de leur famille, de leur peuple, de leur nation. Au-delà des diversités culturelles et personnelles semble exister un point impersonnel, inactuel et anachronique qui fonctionne comme ce lieu d'où peuvent s'opérer l'interprétation, la traduction et une certaine compréhension des différentes expériences culturelles et individuelles. En ce point, émerge le sentiment qu'entendre, interpréter et transposer sont d'autant plus possible que chacune de ces expériences n'est en réalité qu'une tentative de donner un destin à cette communauté originaire d'affect qui relève de l'informe, de l'infantile, de l'inaccompli et qui s'exprime avant tout comme une aspiration au devenir.

À entendre une multitude de vies, le psychanalyste éprouve le sentiment qu'il y a une origine commune à toutes ces figures du destin. Plus d'une fois, l'individu confronté à une rupture traumatique du pare-excitation se tourne, lorsqu'il perd l'exercice de ses moyens et les résultats psychiques qu'il avait déjà acquis, vers des solutions auxquelles ont déjà recouru, autrefois, les ancêtres de l'espèce. Elles sont là comme prêtes à porter, toujours disponibles : un fonds culturel où il peut être puisé pour répondre au plus vite aux urgences de la vie. Les rêves typiques que l'on peut interpréter de manière symbolique témoigneraient de cela.

La récusation de la féminité. Le biologique freudien

Dans toutes les cures, tôt ou tard, il devient évident que le travail d'interprétation ne suffit pas à lever l'amnésie infantile, que le travail de remémoration rencontre un point de butée indépassable. Le travail de la construction permet alors de proposer une histoire qui ne comblera pas la lacune dessinée par les remémorations, mais qui tiendra lieu d'un équivalent de l'oublié en ayant une réelle efficacité thérapeutique. Ce qui nous a toujours frappés tant dans les constructions de Freud que dans celles des collègues et dans les nôtres, c'est la forme qu'elles prennent. Elles s'appuient sur quelques éléments propres 
à l'histoire du patient qu'elles intègrent dans un récit qui a souvent une allure de généralité et une dimension universelle : «J'ai toujours su qu'un jour naîtrait un enfant qui s'appellerait $X$, qui aimerait sa maman et qui souhaiterait la disparition de son papa », " quand vous aviez tel âge, votre maman a attendu un enfant, vous vous êtes senti abandonné et avez ressenti une violente colère contre votre papa. » Tous ces éléments ne prouvent indubitablement rien, mais ils concourent tout de même à apporter quelques fondements à l'idée de schèmes phylogénétiques. Nous sommes là devant les manifestations résiduelles de la cure "qui demandent à être dérivées de la phylogenèse » (Freud, 1939 a, p. 207).

Mais ce sont surtout les autres manifestations résiduelles que sont le sentiment inconscient de culpabilité, le masochisme originaire, la réaction thérapeutique négative, l'excessivité du facteur quantitatif, l'attachement aux bénéfices secondaires, l'autocratisme de la personne qui nous semble constituer les arguments déterminants en faveur de l'hypothèse controversée. En elles, s'exprime ce que L'Analyse finie et l'Analyse infinie désigne comme le roc d'origine sur lequel échoue le pouvoir de l'analyse. Elles sont ces parties qui restent à la traîne du travail psychique et dont l'existence se comprend dès lors que l'on se rappelle que, dans le cours du développement, aucune phase antérieure n'est surmontée sans laisser de traces. Il subsiste toujours des restes non dépassés (voire non dépassables) de la précédente organisation dans celle qui lui succède. Mais qu'il en soit ainsi ne fait que mettre en lumière deux données structurelles de la vie psychique : une partie des pulsions n'est pas utilisable pour les fins du traitement psychique et rien ne saurait faire disparaître les traces agissantes de ce qui est une fois venu à la vie. La Vatersehnsucht serait l'une des premières, voire la première manifestation résiduelle, la trace de ce qui, lors du renoncement à la satisfaction d'où naît le psychique, n'a pas pu être traité par ce dernier. Elle agit depuis inlassablement en tant que noyau du psychique qui ne cesse de faire retour sur le devant de la scène et qui, ne pouvant être accueilli entièrement, ne cesse de donner lieu à des motions substitutives déformées et aux réactions qui en découlent. C'est le roc d'origine tel que nous le présente L'Analyse finie et l'Analyse infinie qui accrédite le plus l'existence de l'héritage archaïque et le fait qu'en son cœur œuvre la Vatersehnsucht. Ce qui est avancé est que, dans toutes les cures, l'analyse rencontre, de la part des deux sexes, une récusation de la féminité qui se présente comme un obstacle à la cure. En ce point de résistance, surgit le sentiment que l'on n'a aucune prise sur elle et qu'on ne peut plus escompter le moindre changement de la part du patient. Cette résistance prend la forme soit d'un souhait de pénis, soit d'une rébellion contre la position passive. La première forme prédomine plutôt chez la 
femme, la seconde chez l'homme, mais toutes deux sont présentes dans les deux sexes. À l'origine, il y a quelque chose qui est commun aux deux sexes qui ne connaîtra un destin différent qu'à partir de la signification psychique qui sera donnée à la différence anatomique plus tard sous l'influence de l'éducation et de la culture.

\section{La Vatersehnsucht, le roc d'origine}

Ce qui fait obstacle à la cure touche à la relation que l'humain qu'il soit homme ou femme entretient avec le père. Pour la psychanalyse, les positions féminine et masculine n'ont pas à être entendues depuis la seule différence anatomique, mais davantage comme l'alternative de positionnement par rapport au père devant laquelle se trouve placé, à l'orée de sa vie et tout au long de celle-ci, chaque humain et du choix qu'il est susceptible de faire. Le souhait de pénis comme la rébellion contre la position passive ne peuvent se comprendre qu'en précisant qu'il s'agit du pénis du père et d'un refus de la passivité devant le père. Sans cette précision, nous tomberions dans l'erreur des théories d'Adler bâties sur le complexe d'infériorité, sa compensation et la protestation virile. Ce qui se rencontre à la fin de la cure, c'est à quel point la Vatersehnsucht nous constitue, nous détermine et reste agissante. À notre origine, l'exigence drastique d'une pulsion qui exige de parvenir à ses fins immédiatement, sans retenue et sans reste, une pulsion qui ne vise que l'assouvissement qui la ferait s'éteindre et supprimerait toutes les raisons d'insatisfaction qui la font perdurer. Nous reconnaissons là, la pulsion de mort dans laquelle nous proposerons de voir un pendant dans le monde interne des caractéristiques qui définissent le père de la horde primitive : la pulsion de mort serait une " intériorisation » de ce père, c'est ainsi qu'il existerait en chacun dès le départ.

Je proposerai d'envisager que le nouveau-né est, en vérité historique et en réalité psychique, la réincarnation du père de la horde. Il l'est pendant tout ce temps qui précède la survenue de l'Hilflosigkeit. Le bébé est impitoyable ${ }^{39}$, comme l'était le père de la horde, il l'est sans haine et sans sadisme, car ces deux sentiments impliquent une intentionnalité qui n'existe pas en cet état originaire où il n'y a qu'un but : la satisfaction. Le nourrisson a un besoin impitoyable de ce qu'il n'a pas encore reconnu comme sa mère. Sous l'emprise de celui-ci, il ne s'intéresse pas aux effets de son amour instinctif et il n'est pas encore conscient de ce désir dont l'inassouvissement deviendra le moteur de son histoire. Cet état sera modifié à la suite de l'expérience de satisfaction rendue possible par la

39. Je fais référence à ce que Winnicott a décrit sous le nom d'amour impitoyable. 
modification du monde extérieur résultant de l'intervention d'une aide étrangère. Et nous retrouvons la séquence des états mélancolique et maniaque consécutifs au meurtre du père et au recul des meurtriers. Imaginer le nourrisson comme étant une réactualisation in vivo et in prcesentia du père de la horde fait entendre que cette histoire ne concerne pas seulement les petits garçons, mais aussi les petites filles. La question du père est au fondement de l'organisation de l'Edipe en complexe, mais elle lui préexiste logiquement et elle éclaire autrement la question de la bisexualité comme ressource constitutive des êtres humains. Le travail psychique permettrait-il, en accueillant davantage cette bisexualité, d'échapper à la récusation du féminin à l'œuvre dans les deux sexes? Et, si tel était le cas, cela ne représenterait-il pas un pas vers un affranchissement du père ?

Dès le début, donc, cette aspiration à un assouvissement total commune à tous les humains quel que soit leur sexe est représentée dans le mythe freudien par le désir et le droit de posséder sexuellement la mère pour soi tout seul. Si l'identification primaire porte sur le père, c'est parce la pulsion pousse à être comme le père de la préhistoire pour posséder exclusivement la mère.

Le souhait de pénis du père est dans un premier temps commun aux deux sexes et il est souhait d'être ce pénis dont est porteur celui qui possède pleinement la mère. Ce souhait connaîtra avec le temps des dérivations influencées et par l'anatomie, et par la culture. La petite fille s'identifiera secondairement à l'objet désiré et voudra, à défaut de posséder la mère, être possédée par celui qui l'a possédée effectivement. Le garçon, constatant qu'il ne peut prendre effectivement la place du père en conclura que, s'il en est ainsi, c'est, sans doute, parce que le père était le plus fort et que son pénis était plus grand. Il s'engagera dans cette rivalité masculine où il s'épuise à comparer la taille des pénis, plutôt que d'avoir à envisager qu'il a reculé sur son désir de prendre la place du père en espérant pouvoir ainsi éviter la mise à mort.

La rébellion est contre celui qui, possédant la mère, nous barre la route qui conduirait à la pleine satisfaction de nos souhaits. Elle est commune originairement aux deux sexes. Si elle agit de manière prédominante chez le garçon, c'est parce que celui-ci n'aura pas à procéder en grandissant à un changement du choix d'objet. Mais la rébellion, en revanche, se verra renforcée durablement par la crainte de la castration et la force du complexe qui en découlera.

Avec le refus de la féminité entendue comme refus de la passivité et de la soumission au père, nous disposons d'un levier pour la compréhension psychanalytique des obstacles insurmontables que rencontre le travail de la cure et de la fragilité du travail de culture. À la fin de la cure et au cœur de la culture, nous retrouvons sans cesse ce refus et la rébellion qui l'exprime noués paradoxalement à une attente croyante d'une totale satisfaction qui va se constituer en irrépressible besoin de croire. Celui-ci ne tarde, semble-t-il, 
jamais longtemps à trouver dans les accidents de la vie les matériaux auxquels se fixera l'individu en devenant avec une facilité déconcertante un croyant ou un militant aveugle et sourd à tout ce qui n'est pas sa croyance ou sa cause. Et il sera prêt non seulement à se sacrifier pour elles, mais à éliminer ceux qui n'y adhéreraient pas ou ceux dont l'existence les remettrait en cause.

AFFRANCHISSEMENT DE L'ÊTRE... EN SOUFFRANCE

Je pourrais considérer que ce sous-titre est la conclusion de ce rapport, mais cela paraîtrait un peu cavalier. Renoncer au père et s'affranchir de son autorité font bien partie des fins de la psychanalyse, mais la partie est loin d'être gagnée et nous ne savons pas si nous pouvons vraiment espérer l'atteindre. Si la Vatersehnsucht est effectivement, comme nous le pensons avec Freud, le cœur et le moteur de l'âme humaine et qu'elle appartient à quelque chose de l'ordre d'un héritage archaïque, la seule possibilité de parvenir davantage aux fins de la psychanalyse réside dans l'hypothèse que les traces du phylogénétique en nous sont modifiables au cours de l'ontogenèse. Si l'héritage archaïque peut être transformé par le truchement de l'accidentel dont fait partie la cure, alors affranchissement et renoncement deviendraient des perspectives moins idéalistes et plus réalistes. Cela signifierait que, lors du processus du retour du refoulé, celui-ci pourrait connaître un autre destin que celui de la religiosité. Mais même si cela s'avérait possible plus fréquemment que cela ne semble avoir été le cas dans l'histoire de l'espèce et dans l'histoire individuelle, la modification de l'héritage archaïque n'abolirait pas l'interrogation sur la dimension constitutionnelle. Les limites rencontrées par le travail de la cure et par le travail de culture ne seraient plus exactement les mêmes, mais cela ne signifierait pas qu'il n'y a plus de limites inhérentes au psychique. Dans notre pratique quotidienne, nous devrons continuer à nous interroger humblement sur ce qui est et ne peut pas être autrement, et sur ce qui est mais peut tout aussi bien être autrement, et veiller à ne pas nous dérober à cette question en nous prenant pour un sauveur, pour un pédagogue, pour un consolateur. Le succès de la cure psychanalytique est atteint lorsque qu'elle a renforcé chez le patient cet amour de la vérité, entendu comme reconnaissance de la dimension de l'Anankè, qui lui permet de supporter les efforts pour aller audelà du principe de plaisir et affronter l'état réel du monde.

François Villa 30 boulevard de Strasbourg 75010 Paris villa@univ-paris-diderot.fr 


\section{RÉFÉRENCES BIBLIOGRAPHIQUES}

Adorno T., Minima Moralia. Réflexions sur la vie mutilée, Paris, Payot, 1980.

Andréas-Salomé L. (1928), Ce qui découle du fait que ce n'est pas la femme qui a tué le père, L'Amour du narcissisme : textes psychanalytiques, Paris, Gallimard, 1980.

Assmann J., Moïse l'égyptien, Paris, Aubier, 2001.

Assoun P.-L., Fonctions freudiennes du père, Le Père. Métaphore paternelle et fonctions du père, l'Interdit, la Filiation, la Transmission, Paris, Denoël, 1989.

Balestrière L., Freud et la question des origines, Bruxelles, De Boeck, 2008.

Barriel V. (1997), L'ADN fossile, http://www.cnrs.fr/cw/dossiers/dosevol/decouv/articles/ chap7/barriel.html.

Beetschen A. (2003), L'accomplissement et l'atteinte, Revue française de psychanalyse, t. LXVII, $\mathrm{n}^{\circ} 5$.

Benslama F. (2004), D'un renoncement au père, Topique, no 85.

Benslama F., Transfiguration du père, in Lauru D. et Le Run J.-L., Figures du père à l'adolescence, Ramonville-Saint-Agne, Érès, 2004.

Benveniste E., Le Vocabulaire des institutions indo-européennes, 1, Paris, Minuit, 1969.

Braunschweig D. (1991), Fantasmes originaires et Surmoi : la phylogenèse, Revue française de psychanalyse, $\mathrm{t}$. LV, $\mathrm{n}^{\circ} 5$.

Certeau M. de, L'Écriture de l'histoire, Paris, Gallimard, 1975.

Darwin Ch. (1872), L'Expression des émotions chez l'homme et les animaux, Paris, Édition C.T.H.S., 1998.

Darwin Ch. (1891), La Filiation de l'homme et la sélection liée au sexe, Genève, Slatkine, 2012.

Derrida J., L'Écriture et la Différence, Paris, Le Seuil, 1967.

Derrida J., Mal d'Archive, Paris, Galilée, 1995.

Descola Ph., Par-delà nature et culture, Paris, Gallimard, 2005.

Donnet J.-L., Surmoi, Paris, Puf, « Monographie de la Revue française de psychanalyse $», 1995$.

Donnet J.-L., L'Humour et la Honte, Paris, Puf, 2009.

Dor J., Le Père et sa fonction en psychanalyse, Ramonville-Saint-Agne, Érès, 1998.

Douville O., Lévi-Strauss et le mythe freudien du père, in Drach M. et Toboul B., L'Anthropologie de Lévi-Strauss et la psychanalyse. D'une structure l'autre, Paris, La Découverte, 2008.

Fédida P., Crise et contre-transfert, Paris, Puf, 1992.

Fédida P., Le Site de l'étranger, Paris, Puf, 1995.

Fédida P., Widlocher, D. (dir.), Les Évolutions : phylogenèse de l'individuation, Paris, Puf, 1994.

Ferenczi S. (1934), Le problème de la fin de l'analyse, Psychanalyse, 4, Paris, Payot, 1982.

Ferenczi S. (1934), Réflexions sur le traumatisme, Psychanalyse, 4, Paris, Payot, 1982.

Florence J., L'Identification dans la théorie freudienne, Bruxelles, Facultés Universitaires Saint Louis, 1978.

Freud S. (1895), Projet d'une psychologie, Lettres à Wilhelm Fliess 1887-1904, Paris, Puf. 
Freud S. (1900 a [1899]), L'interprétation du rêve, OCF.P, IV, Paris, Puf, 2003.

Freud S. (1905 d), Trois essais sur la théorie sexuelle, ocF.P, VI, Paris, Puf, 2006.

Freud S. $(1910$ c), Un souvenir d'enfance de Léonard de Vinci, OCF.P, X, Paris, Puf, 1993.

Freud S. (1911 b), Formulations sur les deux principes de l'advenir psychique, OCF.P, XI, Paris, Puf, 1998.

Freud S. (1911 c), Remarques psychanalytiques sur l'autobiographie d'un cas de paranoïa (le Président Schreber), OCF.P, X, Paris, Puf, 1993.

Freud S. (1912-1913 a), Totem et Tabou, ocF.P, XI, Paris, Puf, 1998.

Freud S. (1914 c), Pour introduire le narcissisme, La Vie sexuelle, Paris, Puf, 1969 ; ocF.P, XII, 2005.

Freud S. (1915 b), Considérations actuelles sur la guerre et sur la mort, Essais de psychanalyse, Paris, Payot, 1989 ; OCF.P, XIII, Paris, Puf, 1988.

Freud S. (1916-1917 a [1915-1917]), Conférences d'introduction à la psychanalyse, OCF.P, XIV, Paris, Puf, 2000.

Freud S. (1918 b [1914]), À partir de l'histoire d'une névrose infantile, OCF.P, XIII, Paris, Puf, 1988.

Freud S. $(1919 d)$, Introduction à « La psychanalyse des névrosés de guerre », OCF.P, XV, Paris, Puf, 1996.

Freud S. (1919 g), Avant-propos à Theodor Reik : «Problèmes de psychologie religieuse », OCF.P, XV, Paris, Puf, 1996.

Freud S. (1920 g), Au-delà du principe de plaisir, Essais de psychanalyse, OCF.P, XV, Paris, Puf, 1996.

Freud S. (1921 c), Psychologie collective et analyse du Moi, OCF.P, XVI, Paris, Puf, 1991.

Freud S. (1923 b), Le Moi et le Ça, OCF.P, XVI, Paris, Puf, 1991.

Freud S. (1924 e), La perte de la réalité dans la névrose et la psychose, OCF.P, XVII, Paris, Puf, 1992.

Freud S. (1937 c), L'analyse avec fin et l'analyse sans fin, OCF.P, XX, Paris, Puf, 2010.

Freud S. (1937 d), Constructions dans l'analyse, OCF.P, XX, Paris, Puf, 2010.

Freud S. (1939 a), L’Homme Moïse et la religion monothéiste, oCF.P, XX, Paris, Puf, 2010.

Freud S. (1940 a [1938]), Abrégé de psychanalyse, OCF.P, XX, Paris, Puf, 2010.

Freud S. (1940 e [1938]), Le clivage du Moi dans le processus de défense, OCF.P, XX, Paris, Puf, 2010.

Freud S. (1960 a [1873-1939]), Correspondance 1873-1939, Paris, Gallimard, 1966.

Freud S. (1985 a [1915]), Vue d'ensemble sur les névroses de transfert. Un essai métapsychologique, OCF.P, XIII, Paris, Puf, 1988.

Freud S. (1985 c), Lettres à Wilhelm Fliess : 1887-1904, Paris, Puf, 2006.

Freud S., Binswanger L., Correspondance 1908-1938, Paris, Calmann-Lévy, 1995.

Freud S., Breuer J., Etudes sur l'hystérie, Paris, Puf, 1978.

Freud S., Ferenczi S., Correspondance, 1908-1914, Paris, Calmann-Lévy, 1992.

Freud S., Zweig A., Correspondance 1927-1939, Paris, Gallimard, 1973. 
Gantheret F., Préface à Totem et Tabou, in Freud S., Totem et Tabou, Paris, Gallimard, 1993.

Girard M. (2012), Expériences de la communauté. Hétérologies politiques de Georges Bataille, Université Paris 8 - Vincennes Saint-Denis.

Godelier M., Hassoun J., Meurtre du père, sacrifice de la sexualité, Strasbourg, Arcanes, 1996.

Godelier M., Au fondement des sociétés humaines, Paris, Albin Michel, 2007.

Goldenweiser A. (1910), Totemism, an Analytical Study, The Journal of American Folklore, n' 23.

Goldstein B., Reinscribing Moses, Cambridge, Massachussets and London England, Harvard University Press, 1992.

Gould S. J., Ontogeny and Phylogeny, Cambridge, Massachussets, London, England, Harvard University Press, 1977.

Granoff W., Filiations, Paris, Minuit, 1975.

Green A., Le Travail du négatif, Paris, Minuit, 1993.

Green A., Le Discours vivant. La conception psychanalytique de l'affect, Paris, Puf, 2004.

Gribinski M., Dans un monde qui ne veut rien, in Cyssau C., Villa F., La Nature humaine à l'épreuve de Winnicott, Paris, Puf, 2006.

Guillaumin J. (2000), Archéologie du père, entre l'angoisse d'une présence et la métaphore d'une absence, le « père de la préhistoire personnelle », Topique, $\mathrm{n}^{\circ} 72$.

Guillaumin J., Roger G. (dir.), Le Père : figures et réalité, Bordeaux, L’Esprit du Temps, 2003.

Héritier F., Les Deux sœurs et leur mère. Anthropologie de l'inceste, Paris, Odile Jacob, 1993.

Huxley J., Evolution in Action, New York, Harper, 1953.

Jablonka E., Lamb Marion J. (eds) (2005), Evolution in Four Dimensions: Genetic, Epigenetic, Behavioral, and Symbolic Variation in the History of Life, MIT Press.

Jones E., La Vie et l'Euvre de Sigmund Freud, III, Les dernières années 1919-1939, trad. franç. L. Flournoy, Paris, Puf, 2006.

Juillerat B. (2000), L'atome de parenté est-il soluble dans la psychanalyse ?, Topique, $\mathrm{n}^{\circ} 75$.

Kahn L. (2005), Quand la Shoah est un trauma et que le père disparait de la théorie analytique, Penser/rêver, $\mathrm{n}^{\circ} 7$.

Kahn L., Faire parler le destin, Paris, Klincksieck, 2005.

Kahn L. (2009), Un père, un fils, on mange, Penser/rêver, 16.

Krœber A. L. (1920), Totem et tabou : une psychanalyse ethnologique, Revue française de psychanalyse, t. LVII, n 3, 1993.

Krœber A. L. (1924), Totem et tabou : 1'après-coup, Revue française de psychanalyse, t. LVII, no 3, 1993.

La Boétie E. (de) (1576), Discours de la servitude volontaire, Paris, Flammarion, 1993.

Lacan J. (1956-1957), Le Séminaire IV : La relation d'objet, Paris, Le Seuil, 1994. 
Lacan J. (1969-1970), Le Séminaire XVII : l'envers de la psychanalyse, Paris, Le Seuil, 1991.

Lacan J. (1972-1973), Le Séminaire XX : Encore, Paris, Le Seuil, 1975.

Lacan J., Des noms du père, Paris, Le Seuil, 2005.

Lacoste P. (1985), Le manuscrit retrouvé : une lecture : Freud, Übersicht der Übertragungsneurosen, présenté et commenté par Ilse Grubrich-Simitis, S. Fischer, Nouvelle revue de Psychanalyse, $\mathrm{n}^{\circ}$ 32, Paris, Gallimard.

Laplanche J., Hölderlin et la question du père, Paris, Puf, 1961.

Laplanche J. (1998), La psychanalyse : mythe et théorie, Revue française de psychanalyse, t. LXII, $\mathrm{n}^{\circ} 3$.

Le Bloc-notes de la Psychanalyse (1995), nº 13, Le père.

Lemaigre B. (1984), Meurtre du père et mise à l'écart du féminin, Psychanalystes, $\mathrm{n}^{\circ} 13$.

Lemaigre B. (1988), Le travail de la phylogénèse dans l'œuvre de Freud, Psychanalystes, $\mathrm{n}^{\circ} 26$.

Lévi G., Les historiens, la psychanalyse et la vérité, in Abel O., Castelli-Gattinara E., Loriga S., Ullern-Weité I., La Juste Mémoire. Lectures autour de Paul Ricœeur, Paris, Labor et Fides, 2006.

Lévi-Strauss Cl., Le Totémisme aujourd'hui, Paris, Puf, 1962.

Lévi-Strauss Cl., Les Structures élémentaires de la parenté, Paris, Mouton, 1967.

Lewis R., Barisse R., Pourquoi j'ai mangé mon père, Arles, Actes Sud, 1999.

L'inconscient, $\mathrm{n}^{\circ} 5$, La paternité.

Loraux N. (1991), L'Homme Moïse et l'audace d'être historien, Le cheval de Troie, $\mathrm{n}^{\circ} 3$.

Louppe A. (2012), Edipe en instances, Revue française de psychanalyse, t. LXXVI, $\mathrm{n}^{\circ} 5$.

Malamoud Ch., L'apport freudien à l'histoire des religions, in Kaufman P., L'Apport freudien, Paris, Bordas, 1993.

Matthews S.G. and Phillips D.I.W. (2010), Minireview: Transgenerational Inheritance of the Stress Response : A New Frontier in Stress Research, Endocrinology, vol. 151, $\mathrm{n}^{\circ} 1$.

Mauss Marcel (1896), Le totémisme selon Frazer et Durkheim, Euvres, I, 1970.

Mérot P. (2011), Traces du maternel dans le religieux, Revue française de psychanalyse, t. LXXV, no 5 .

Moscovici M., Préface de Freud S., L'Homme Moïse et la religion monothéiste, Paris, Gallimard, 1986.

Moscovici M., Il est arrivé quelque chose. Approches de l'évènement psychique, Paris, Payot, 1991.

Moscovici M. (1993), Les préhistoires : pour aborder Totem et Tabou, Revue française de psychanalyse, t. LVII, $\mathrm{n}^{\circ} 3$.

Nietzche F., Gai savoir, Euvres complètes, Paris, Gallimard, 1982.

Nunberg H., Federn E., Les Premiers Psychanalystes : minutes de la Société psychanalytique de Vienne, III, Paris, Gallimard, 1976.

Ody M., Edipe comme attracteur, La psychanalyse : questions pour demain, Paris, Puf, « Monographies de la Revue française de psychanalyse », 1990. 
Pulman B. (1991), Les anthropologues face à la psychanalyse : premières réactions et Ernest Jones et l'anthropologie, Revue internationale d'histoire de la psychanalyse, IV.

Ritvo L. B., L'Ascendant de Darwin sur Freud, Paris, Gallimard, 1990.

Rosolato G., Essais sur le symbolique, Paris, Gallimard, 1969.

Schneider M., Généalogie du masculin, Paris, Aubier, 2000.

Séchaud E. (2005), Perdre, sublimer, Revue française de psychanalyse, t. LXIX, $\mathrm{n}^{\circ} 5$.

Stoloff J.-C., La Fonction paternelle, Paris, In Press, 2007.

Stein C. (1968), Le père mortel et le père immortel, L'inconscient, $\mathrm{n}^{\circ} 5$.

Sulloway F.J., Freud, biologiste de l'esprit, Paris, Fayard, 1981.

Topique (2000), $\mathrm{n}^{\circ} 72$, La fonction paternelle.

Tort P. (sous la dir. de), Dictionnaire du darwinisme et de l'évolution, Paris, Puf, 1996.

Tort M., La Fin du dogme paternel, Paris, Aubier, 2005.

Vanier A. (2001), Homme/père, Figures de la psychanalyse, $\mathrm{n}^{\circ} 5$.

Vanier A., Novalis, l'écriture et le nom, in Sayer F. (ed.), La Littérature et le Divan. L'écrivain face au psychanalyste, Paris, Hermann, 2011.

Villa F., L'exigence technique paradoxale dont répond la construction en analyse, in Fédida P., Villa F., Le cas en controverse, Paris, Puf, « Monographies de psychopathologie $», 1999$.

Villa F. (2000), La théorie sexuelle de l'acte. Préliminaires d'une théorie de l'action psychothérapique, Thèse de doctorat, Université Paris Diderot-Paris 7.

Villa F., La notion de caractère chez Freud, Paris, Puf, 2009 a.

Villa F. (2009 b), Au cœur du rêve, la horde, Penser/rêver, n 15, Toute-puissance.

Villa F., Malaise dans la civilisation et désastre totalitaire, L'annuel de l'Association Psychanalytique de France, Paris, Puf, 2011.

Villa F., Weil E., Lettre à Nathalie... l'absente, in Lévy G., L'Esprit d'insoumission. Réflexions autour de la pensée de Nathalie Zaltzman, Paris, Campagne Première, 2011.

Waddington C.H. , How animals develop, Londres, George Allen \& Unwin Ltd, 1946.

Weber M., L'Éthique protestante et l'esprit du capitalisme (1904-1905), trad. franç. J. Chavy, Paris, Plon, 1964.

Weil E. (2000), Silence et latence, Revue française de psychanalyse, t. LXIV, $\mathrm{n}^{\circ} 1$.

Weil E., Isaac Bashevis Singer: reconstructeur d'identités collectives perdues?, in Szurek J.-Ch., Wieviorka A., Juifs et Polonais, 1939-2008, Paris, Albin Michel, 2009.

Weitzman J. (2011), Epigenetics : Beyond face value, Nature, no 477.

Widlöcher D., préface de Correspondance de Sigmund Freud avec le pasteur Pfister, 1909-1939, Paris, Gallimard, 1966.

Winnicott D.W., La Nature humaine, trad. franç. B. Weil, Paris, Gallimard, 1990.

Yerushalmi Y. H., Le Mö̈se de Freud. Judä̈sme terminable et interminable, Paris, Gallimard, 1993.

Zaltzman N., De la guérison psychanalytique, Paris, 1998.

Zaltzman N., L'Esprit du mal, Paris, L'Olivier, 2007. 